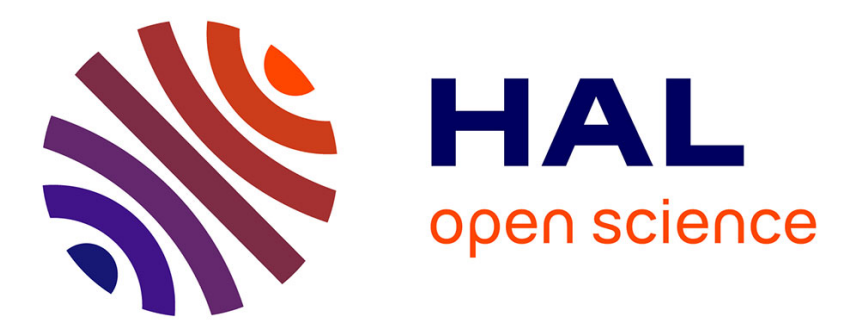

\title{
Blind filters based on envelope spectrum sparsity indicators for bearing and gear vibration-based condition monitoring
}

Cédric Peeters, Jérôme Antoni, Jan Helsen

\section{To cite this version:}

Cédric Peeters, Jérôme Antoni, Jan Helsen. Blind filters based on envelope spectrum sparsity indicators for bearing and gear vibration-based condition monitoring. Mechanical Systems and Signal Processing, 2020, 138, pp.106556. 10.1016/j.ymssp.2019.106556 . hal-03211956

\author{
HAL Id: hal-03211956 \\ https://hal.science/hal-03211956
}

Submitted on 3 Sep 2021

HAL is a multi-disciplinary open access archive for the deposit and dissemination of scientific research documents, whether they are published or not. The documents may come from teaching and research institutions in France or abroad, or from public or private research centers.
L'archive ouverte pluridisciplinaire HAL, est destinée au dépôt et à la diffusion de documents scientifiques de niveau recherche, publiés ou non, émanant des établissements d'enseignement et de recherche français ou étrangers, des laboratoires publics ou privés. 


\title{
Blind filters based on envelope spectrum sparsity indicators for bearing and gear vibration-based condition monitoring
}

\author{
Cédric Peeters $^{\mathrm{a}, *}$, Jérôme Antoni ${ }^{\mathrm{b}}$, Jan Helsen ${ }^{\mathrm{a}}$ \\ ${ }^{a}$ Vrije Universiteit Brussel, Department of Mechanical Engineering, Pleinlaan 2, Elsene, Belgium \\ ${ }^{\mathrm{b}}$ Univ Lyon, INSA-Lyon, Laboratoire Vibrations, F-69621 Villeurbanne, France
}

\section{A R T I C L E I N F O}

\section{Article history:}

Received 3 May 2019

Received in revised form 4 October 2019

Accepted 5 December 2019

\section{Keywords:}

Blind filter

Sparsity

Fault detection

Envelope spectrum

Rayleigh quotient iteration

Condition monitoring

\begin{abstract}
A B S T R A C T
This paper investigates a novel perspective on blind filtering of vibration signals with the purpose of fault detection in rotating machinery. Instead of maximizing a property of the time-domain signal such as kurtosis to find an optimal filter, the sparsity of its envelope spectrum is maximized. The underlying assumption for this approach is that faults of rotating components such as bearings introduce second-order cyclostationary content into the signal. This cyclostationary content manifests itself as discrete peaks in the envelope spectrum (given the speed is stationary). These peaks thus increase the sparsity of the envelope spectrum as a consequence. Therefore this paper derives blind filter formulations that try to filter out a signal with the most sparse envelope spectrum. Blind filters are derived using three different sparsity measures, i.c. $\frac{l_{2}}{l_{1}}$-norm, Hoyer Index, and spectral negentropy. Key in the iterative optimization procedure is the usage of the Rayleigh quotient to update the filter coefficients. One major advantage of this approach is that no prior information about characteristic fault frequencies of the mechanical components of interest needs to be known. Inspection of simulation and experimental results shows that the proposed approach is a simple yet effective way of tracking faults with a cyclostationary signature.
\end{abstract}

(c) 2019 Elsevier Ltd. All rights reserved.

\section{Introduction}

Condition monitoring of rotating machinery is becoming increasingly popular and advanced. Industry starts to incorporate more and more dedicated condition monitoring systems in their design. Not only is the industry looking to include basic condition monitoring as a potential service, also general interest in more advanced monitoring tools is growing thanks to the ever-improving computational power, expanding storage capabilities, and decreasing cost of modern day IT systems. Despite this trend, it sometimes remains difficult to implement some of the more advanced methods due to incomplete knowledge about the system of interest. Complex machines can consist of dozens of bearings and gears, modern examples are gearboxes of wind turbines and helicopters. These machines typically have one or multiple planetary gear stages in combination with parallel gear stages. Not all kinematic information about the system is always made available to the machine operator by the manufacturer, or the information might be inaccurate due to reparations with new components. This issue constitutes the need for a method capable of tracking the condition of these components without the need for a priori knowledge about the kinematics.

\footnotetext{
* Corresponding author.

E-mail addresses: cedric.peeters@vub.be (C. Peeters), jerome.antoni@insa-lyon.fr (J. Antoni), jan.helsen@vub.be (J. Helsen).
} 
Current practice in condition monitoring systems often revolves around tracking time-domain statistical indicators and more component-specific frequency-domain indicators [1-3]. The advantage of using simple scalar time-domain indicators is that no a priori knowledge about the characteristic fault frequencies is required and thus the number and complexity of the components is not taken into account. This simplifies the analysis procedure significantly, with the trade-off being that it does not allow pinpointing which component is exhibiting the anomalous behavior. Not having any knowledge about the exact location of a fault is a downside, but in an early analysis stage it is often easier and quicker to have a straightforward overview of which measurement sensor is picking up faulty behavior. Afterwards, a more in-depth frequency-based analysis can investigate further and try to determine the missing failure information.

The concept of tracking signal characteristics blindly is already widely employed in industry thanks to the simplicity of time-domain statistical indicators. Nevertheless there is still room to improve further on this blind signal analysis concept and to bring it better in line with other state-of-the-art vibration analysis methods. One of the most popular approaches for fault detection is to look at the cyclostationary behavior of the vibration signal [4-12]. Inspired by this fact, this paper investigates the possibility to utilize the cyclostationary content of a signal in a blind manner. Therefore, instead of just looking at the statistics of the time waveform, the squared envelope spectrum of the signal is employed as a blind means to gain more information about potential defects. The envelope spectrum is probably the most frequently used tool to inspect the cyclostationary behavior of signals. From experience it is known that most mechanical faults of bearings or gears induce some form of cyclostationary behavior in the observed vibration signals $[7,13,14]$. This phenomenon causes the envelope spectrum to exhibit discrete peaks at the corresponding fault frequencies. These peaks arise from the background noise and thus their amplitude is distinguishable from the spectral noise floor. In other words, most mechanical faults increase the sparsity of the envelope spectrum. Sparsity is a very useful concept that has become a widely used tool across various fields in recent years. The use of sparsity (or sparseness) saw a significant increase in interest from scholars thanks to the introduction of compressed sensing in 2004. Candes et al. [15,16] and D. Donoho [17,18] proved that when knowledge is available about the sparsity of a signal, that signal can potentially be reconstructed with even fewer samples than strictly required by the Nyquist-Shannon sampling theorem. This idea is the basis of compressed sensing. Fig. 1 shows how the usage of sparsity increased almost exponentially in research efforts over the last few years, as listed by the Web of Science [19]. This sparsity property can now also be exploited to find a filter that maximizes the sparsity of the envelope spectrum since it can be assumed that this corresponds to a mechanical fault and not to normal behavior.

This paper illustrates the concept of blind filtering using sparsity indicators on the envelope spectrum as a way of tracking the health of bearings. The blind filtering approach is similar to the blind deconvolution idea employed by Minimum Entropy Deconvolution (MED) filtering [20] which uses the kurtosis of the time waveform as the statistic to maximize. MED was

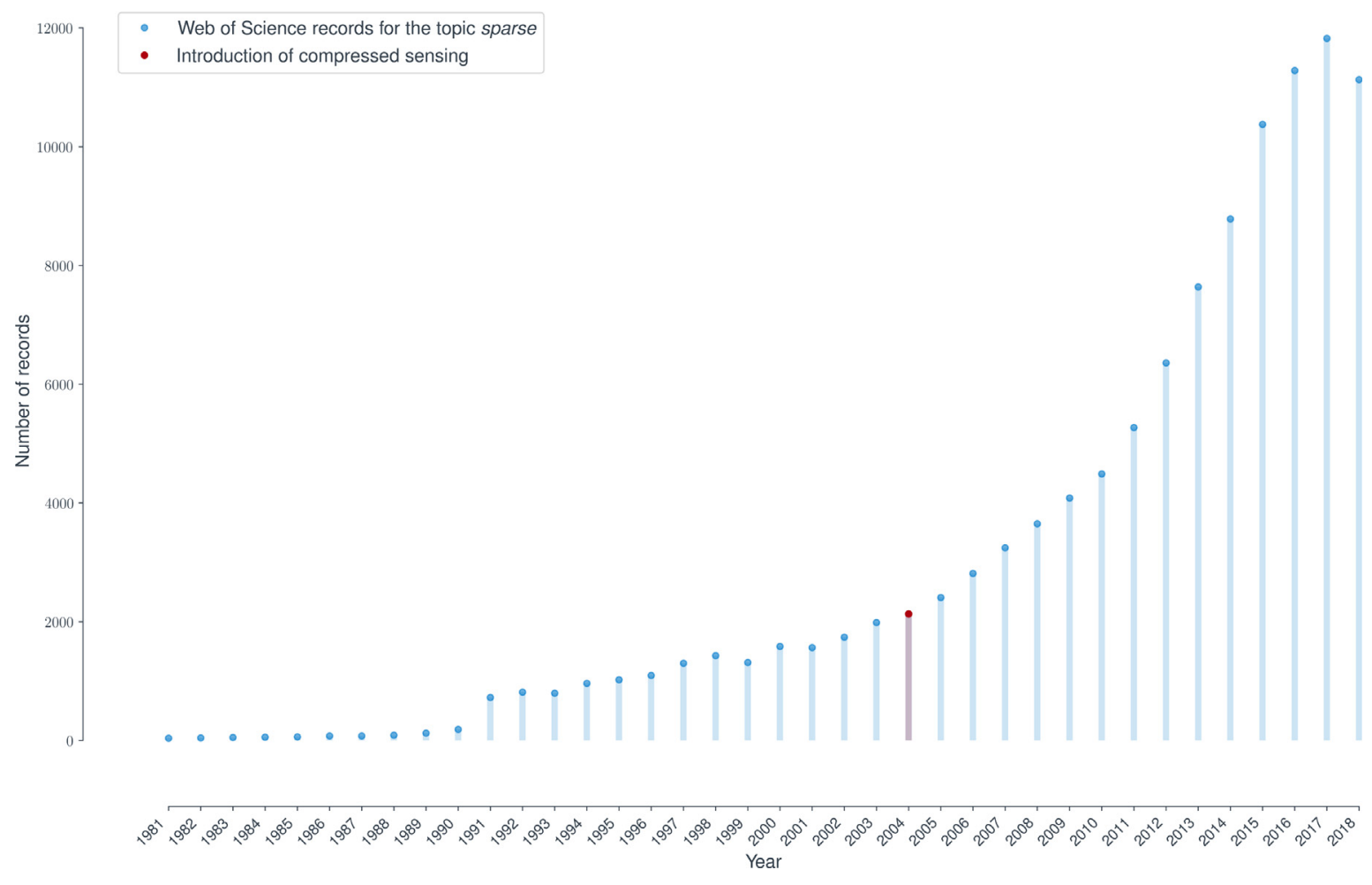

Fig. 1. Evolution of the number of research records in the Web of Science related to the topic of 'sparsity'. 
originally applied in the field of seismic signal processing. Kurtosis however has some disadvantages as a measure to maximize [21]. Kurtosis tends to decrease in value when the frequency of the impulses increases. When the impulses overlap over each other, the kurtosis can even decrease in value to that of a Gaussian signal. On the other hand, when the impulses are so infrequent that there is only one impulse in the measurement, the kurtosis tends to be maximized. This explains why maximizing the kurtosis can lead to erroneous results since it is sensitive to impulsive noise.

Afterwards, improvements and variations on the MED approach were developed. Cabrelli [22] suggested to use the Dnorm (MEDD), an alternative to kurtosis, as a way to find a direct solution to the blind deconvolution problem. Wang et al. reduced the computation time of MEDD by using an adaptive least-squares approach [23]. Broadhead et al. compared the performance of MED and MEDD for varying signal-to-noise ratios (SNR) and found that MEDD outperforms MED in low to moderate SNR. W. Gray [24] analyzed normalized higher-order moments other than kurtosis. McDonald et al. [25] made a comprehensive investigation of MED and its flaws. They afterwards suggest several improvements and extensions to MED in an attempt to circumvent these flaws, such as using multi-point kurtosis instead of standard kurtosis. Obuchowski et al. [26] opted to use the Jarque-Bera statistic, a combination of skewness and kurtosis, for the detection of gear faults. Very often the MED-related techniques get paired with other pre-processing methods, like autoregressive filtering or cepstrum prewhitening, in order to remove deterministic content that could potentially hinder successful deconvolution of the fault signal [2731]. This harmonic removal step can prove useful for the methodology described in this paper as well.

In recent years, there has been a shift in focus from looking at the time waveform statistics to looking at the cyclostationary properties. Maximum correlated kurtosis deconvolution (MKCD) and the multipoint optimal minimum entropy deconvolution (MOMEDA) are technically cyclostationary approaches but were empirically introduced from the perspective of trying to maximize the impulsiveness linked to a certain fault period. Thanks to the recent efforts in further development and promotion of the concept of cyclostationarity for vibration analysis [12,11,32-34], the topic is now a lot more understood and more widely used. A newly proposed approach [35] employs an indicator of second-order cyclostationarity (ICS2) in the process of blind deconvolution. It allows the user to maximize the ICS2 in an efficient and flexible manner thanks to the versatility of the generalized Rayleigh quotient. However, the approach still requires a priori knowledge of the fault frequencies in order to work. To remedy this potential issue, the concept of sparsity can be applied to the envelope spectrum, which is probably the most popular tool to analyze the cyclostationary content of a vibration signal. Wang et al. [36] suggest to use the $\frac{l_{2}}{l_{1}}$-norm on the envelope spectrum of filtered signals. They extend the filterbank idea, originating from the kurtogram, to utilize the $\frac{l_{2}}{l_{1}}-$ norm instead of the kurtosis. The downside of this approach is however that it is still not fully blind in the sense that an end-user still has to inspect the filterbank with the $\frac{l_{2}}{l_{1}}$-norm values and choose the appropriate frequency band. In contrast, the proposed method employs the discussed blind filtering approach such that a suitable filter that maximizes the envelope spectrum sparsity is found automatically. The main "selling" point of the proposed approach as compared to the ICS2 filter is the fact that no a priori information is needed about the characteristic fault frequencies. The proposed method provides a way to track the presence of any modulating fault signature using conventional finite-impulse response filters.

An important remark about the proposed approach is that the blind filtering methodology described in this paper cannot be categorized as blind deconvolution, blind signal separation, or denoising. The proposed approach namely does not attempt to deconvolve the signal in order to recover the source signal (e.g. impulses), nor does it attempt to separate signals from a mixture or remove noise from the signal without distorting it. In fact, it actually does distort the signal such that the envelope spectrum is as sparse as possible. This is a rather new concept since from this perspective the algorithm does not try restoring the signal or recovering the source signals. Instead the algorithm just tries to maximize the figure of merit and thus enhance the envelope spectrum. It is important to take into account this distinction when inspecting filtering results since the results might not correspond to what is expected.

This paper attempts to highlight the utility of sparsity from a cyclostationary perspective and reiterates the versatility of the Rayleigh quotient regarding the indicator choice for the blind filtering step. First, the theoretical background is explained in Section 2. The indicator choice and the derivation of the Rayleigh quotients are described. Next, the method is validated on simulated signals in Section 3 and experimental data of a gearbox trending data set in Section 4. The results show that the proposed approach is capable of extracting a cyclostationary fault signature and that the sparsity measure of the envelope spectrum in itself can be used as a tracking parameter.

\section{Methodology}

To start the optimization of the sparsity of the squared envelope spectrum, a choice needs to be made in which sparsity measure is best employed for this purpose. This point is explained in Section 2.1. Next, an efficient way to find a suitable filter that maximizes the chosen sparsity measure needs to be determined. The concept of blind filtering is explained in Section 2.2.

\subsection{Choice of sparsity measure}

Ever since sparsity became a popular research interest in large part thanks to compressed sensing, many sparsity measures have been introduced. Each one typically has its benefits and disadvantages. A widely used sparsity measure is the 
$\frac{l_{2}}{l_{1}}$-norm, which belongs to the family of $\frac{l_{p}}{l_{q}}$-norms. More than a decade ago, the inverse $\frac{l_{1}}{l_{2}}$-norm (more colloquially known as the Taxicab-Euclidean norm ratio [37]) became much used as a sparsity measure in Non-negative Matrix Factorization [3842]. In fact, it was already being used as a tool for deconvolution in geophysics [24]. This type of norm has recently been used as well in the context of vibration analysis for deconvolution of time-domain waveforms [43]. The $\frac{l_{2}}{l_{1}}$-norm of the envelope spectrum $E_{x}(n)$ of a vector $\mathbf{x}$ is defined as:

$$
\frac{l_{2}}{l_{1}}=\frac{\sqrt{\sum_{n=1}^{N}\left|E_{x}(n)\right|^{2}}}{\sum_{n=1}^{N}\left|E_{x}(n)\right|}
$$

The $\frac{l_{2}}{l_{1}}$-norm is the first out of three sparsity indicators to be investigated in this paper.

Another interesting measure to characterize the squared envelope spectrum is the spectral negentropy in the spectral domain. Spectral negentropy proceeds from interpreting the (square of the) instantaneous energy flow in a signal as a probability distribution. Therefore the definition of negentropy takes into consideration the normalization of the energy flow $\epsilon_{x}$. The spectral negentropy is the negative of spectral entropy and is defined in the time domain as:

$$
\Delta I_{\epsilon}=-H_{\epsilon}=\left\langle\frac{\epsilon_{x}(n)^{2}}{\left\langle\epsilon_{x}(n)^{2}\right\rangle} \ln \left(\frac{\epsilon_{x}(n)^{2}}{\left\langle\epsilon_{x}(n)^{2}\right\rangle}\right)\right\rangle
$$

with $\epsilon_{x}(n)$ the envelope in the time domain of signal $x_{k}(n)$. In the spectral domain this becomes:

$$
\Delta I_{E}=-H_{E}=\left\langle\frac{\left|E_{X}(\alpha)\right|^{2}}{\left\langle\left|E_{x}(\alpha)\right|^{2}\right\rangle} \ln \left(\frac{\left|E_{x}(\alpha)\right|^{2}}{\left\langle\left|E_{x}(\alpha)\right|^{2}\right\rangle}\right)\right\rangle
$$

The negentropy in the time domain quantifies impulsive signal content, much like the kurtogram (Eq. 2 is actually a weighted version of kurtosis). The negentropy in the spectral domain on the other hand quantifies the repetitive or cyclostationary signal content. The negentropy in the spectral domain is therefore investigated as the second measure for blind filtering.

Hurley et al. [41] made a comprehensive comparison of fifteen commonly used sparsity measures. They evaluated these measures using six criteria a desirable measure should possess as an attribute. The Gini Index came out on top, satisfying all six criteria, with the Hoyer Index a close second, satisfying five of the criteria. However, the calculation of the Gini Index requires sorting the values which is difficult to incorporate in the derivation of the blind filter as will become clear later on. Thus, this paper opts to include the Hoyer Index as the third and last sparsity measure of choice for the envelope spectrum. The Hoyer Index was proposed by P. Hoyer [42] in 2004 and is essentially a normalized version of the $\frac{l_{2}}{l_{1}}$-norm. The Hoyer Index evaluates to unity if and only if the spectrum contains only a single non-zero component, and takes a value of zero if and only if all spectral components are equal. It is defined as:

$$
\text { Hoyer Index }=\left(\sqrt{N}-\frac{\sum_{n=1}^{N}|x(n)|}{\sqrt{\sum_{n=1}^{N}|x(n)|^{2}}}\right) \frac{1}{\sqrt{N}-1}
$$

with $x$ being the sample vector, $n$ the sample number, and $N$ the total number of samples.

\subsection{Blind filtering}

The concept of blind filtering is to find a filter that maximizes a certain criterion of the signal starting from a noisy measured signal $\mathbf{x}$ :

$$
\mathbf{s}=\mathbf{X} * \mathbf{h}
$$

where $\mathbf{s}$ is the estimated input, $\mathbf{h}$ is the inverse filter, and $*$ refers to the convolution operation. It should be noted that vectors and matrices are set in bold font to illustrate the difference with scalars. The convolution is expressed as:

$$
\begin{aligned}
& \mathbf{s}=\mathbf{X h} \\
& {\left[\begin{array}{c}
S_{N-1} \\
\vdots \\
x_{L-1}
\end{array}\right]=\left[\begin{array}{ccc}
x_{N-1} & \ldots & x_{0} \\
\vdots & \ddots & \vdots \\
x_{L-1} & \ldots & x_{L-N-2}
\end{array}\right]\left[\begin{array}{c}
h_{0} \\
\vdots \\
h_{N-1}
\end{array}\right]}
\end{aligned}
$$

with $L$ and $N$ the number of samples of $\mathbf{s}$ and $\mathbf{h}$ respectively.

Now the squared envelope $\epsilon_{x}$ can be defined as follows: 


$$
\boldsymbol{\epsilon}_{\boldsymbol{x}}=|\mathbf{s}|^{2}=|\mathbf{X h}|^{2}
$$

It can also be written as:

$$
\boldsymbol{\epsilon}_{x}=\left[\begin{array}{ccc}
s_{0} & \ldots & 0 \\
\vdots & \ddots & \vdots \\
0 & \ldots & s_{L-N+1}
\end{array}\right]^{H} \mathbf{X h}=\operatorname{diag}\left(\mathbf{s}^{\mathbf{H}}\right) \mathbf{X h}
$$

with $\mathbf{s}^{\mathbf{H}}$ being the Hermitian transpose of $\mathbf{s}$, and $\operatorname{diag}\left(\mathbf{s}^{\mathbf{H}}\right)$ being a diagonal matrix with the values of the vector $\mathbf{s}^{\mathbf{H}}$ on its diagonal. The squared envelope spectrum $\mathbf{E}_{\mathbf{x}}$ is then the Fourier transform of this squared envelope:

$$
\mathbf{E}_{\mathbf{x}}=\mathbf{F}^{\mathbf{H}} \boldsymbol{\epsilon}_{x}=\mathbf{F}^{\mathbf{H}} \operatorname{diag}\left(\mathbf{s}^{\mathbf{H}}\right) \mathbf{X h}
$$

with:

$$
\mathbf{F}=\left[\begin{array}{cccccc}
1 & 1 & \ldots & 1 & \ldots & 1 \\
1 & e^{-2 \pi j \frac{1}{L-N}} & \ldots & e^{-2 \pi j \frac{k}{L-N}} & \ldots & e^{-2 \pi j \frac{K-1}{L-N}} \\
\vdots & \vdots & \ddots & & & \vdots \\
1 & e^{-2 \pi j j_{L-N}} & \ldots & e^{-2 \pi j \frac{k n}{L-N}} & \ldots & e^{-2 \pi j \frac{K-1) n}{L-N}} \\
\vdots & \vdots & & & \ddots & \vdots \\
1 & e^{-2 \pi j \frac{L-N-1}{L-N}} & \ldots & e^{-2 \pi j \frac{k(L-N-1)}{L-N}} & \ldots & e^{-2 \pi j \frac{(K-1)(L-N-1)}{L-N}}
\end{array}\right]
$$

with $n=0 . . L-N-1, k=0 . . K-1$ and $K-1$ corresponding to the index of the maximum frequency of interest. $\mathbf{F}$ has dimensions $(L-N, K)$. The defined variables now allow to derive the filters for the three indicators in a fairly efficient manner. The described approach is similar and based on the work presented in [35].

\subsubsection{Derivation of filter using $\frac{l_{2}}{l_{1}}$-norm}

The $\frac{l_{2}}{l_{1}}$-norm of the envelope spectrum $\mathbf{E}_{\mathbf{x}}$ is defined as:

$$
\frac{l_{2}}{l_{1}}=\frac{\sqrt{\sum_{n=1}^{N}\left|E_{x}(n)\right|^{2}}}{\sum_{n=1}^{N}\left|E_{x}(n)\right|}
$$

Rewriting using vector notation, gives:

$$
\frac{l_{2}}{l_{1}}=\frac{\sqrt{\mathbf{E}_{\mathbf{x}}^{H} \mathbf{E}_{\mathbf{x}}}}{\mathbf{E}_{\mathbf{x}}^{H} \operatorname{diag}\left(\frac{1}{\left(\mathbf{E}_{\mathbf{x}}\right)}\right) \mathbf{E}_{\mathbf{x}}}
$$

The denominator is obtained using following identity:

$$
\sum_{n=1}^{N}\left|E_{x}(n)\right|=\sum_{n=1}^{N} \frac{\left|E_{x}(n)\right|^{2}}{\left|E_{x}(n)\right|}=\mathbf{E}_{\mathbf{x}}^{H} \operatorname{diag}\left(\frac{1}{\left|\mathbf{E}_{\mathbf{x}}\right|}\right) \mathbf{E}_{\mathbf{x}}
$$

Manipulating the numerator of Eq. 12 further, it follows that:

$$
\sqrt{\mathbf{E}_{\mathbf{x}}^{H} \mathbf{E}_{\mathbf{x}}}=\frac{\mathbf{E}_{\mathbf{x}}{ }^{H} \mathbf{E}_{\mathbf{x}}}{\sqrt{\mathbf{E}_{\mathbf{x}}^{H} \mathbf{E}_{\mathbf{x}}}}=\mathbf{E}_{\mathbf{x}}^{H} \operatorname{diag}\left(\frac{1}{\sqrt{\mathbf{E}_{\mathbf{x}}^{H} \mathbf{E}_{\mathbf{x}}}}\right) \mathbf{E}_{\mathbf{x}}
$$

Eq. 12 can thus be rewritten in following form:

$$
\frac{l_{2}}{l_{1}}=\frac{\mathbf{E}_{\mathbf{x}}{ }^{H} \operatorname{diag}\left(\frac{1}{{\sqrt{\mathbf{E}_{\mathbf{x}}}}^{H} \mathbf{E}_{\mathbf{x}}}\right) \mathbf{E}_{\mathbf{x}}}{\mathbf{E}_{\mathbf{x}}^{H} \operatorname{diag}\left(\frac{1}{\left|\mathbf{E}_{\mathbf{x}}\right|}\right) \mathbf{E}_{\mathbf{x}}}
$$

Inserting Eq. 9 in Eq. 15 allows to write the ratio in full:

$$
\frac{l_{2}}{l_{1}}=\frac{\mathbf{h}^{\mathbf{H}} \mathbf{X}^{\mathbf{H}} \operatorname{diag}(\mathbf{s}) \mathbf{F} \operatorname{diag}\left(\frac{1}{\sqrt{\mathbb{E}_{\mathbf{x}}^{\mathbf{H}} \mathbf{E}_{\mathbf{x}}}}\right) \mathbf{F}^{\mathbf{H}} \operatorname{diag}\left(\mathbf{s}^{\mathbf{H}}\right) \mathbf{X h}}{\mathbf{h}^{\mathbf{H}} \mathbf{X}^{\mathbf{H}} \operatorname{diag}(\mathbf{s}) \mathbf{F} \operatorname{diag}\left(\frac{1}{\left|\mathbf{E}_{\mathbf{x}}\right|}\right) \mathbf{F}^{\mathbf{H}} \operatorname{diag}\left(\mathbf{s}^{\mathbf{H}}\right) \mathbf{X h}}
$$

The generalized Rayleigh quotient [44] can be recognized in Eq. 16 and can be maximized using an iterative maximization of the eigenvalues: 


$$
\lambda=\frac{\mathbf{h}^{\mathrm{H}} \mathbf{R}_{X W_{1} X} \mathbf{h}}{\mathbf{h}^{\mathrm{H}} \mathbf{R}_{X W_{2} X} \mathbf{h}}
$$

The Rayleigh quotient has the interesting property that its maximal value with respect to $\mathbf{h}$ is equivalent to its largest eigenvalue $\lambda$ and corresponding eigenvector. Thus, maximizing the Rayleigh quotient allows finding the maximal values of the corresponding indicator and filter. In order to obtain real eigenvalues however, the correlation matrices $\mathbf{R}_{X W_{1} X}$ and $\mathbf{R}_{X W_{2} X}$ need to be Hermitian, and $\mathbf{R}_{X W_{2} X}$ needs to be positive semidefinite. If these conditions are met, the Rayleigh quotient offers an efficient means to calculate iteratively the filter coefficients. Only the largest eigenvalue and corresponding eigenvector need to be computed in each iteration, which can be achieved efficiently by using algorithms such as the power method [45].

Thus the $\frac{l_{2}}{l_{1}}$-norm is rewritten as follows:

$$
\frac{l_{2}}{l_{1}}=\frac{\mathbf{h}^{\mathrm{H}} \mathbf{R}_{X W_{1} X} \mathbf{h}}{\mathbf{h}^{\mathrm{H}} \mathbf{R}_{X W_{2} X} \mathbf{h}}
$$

with:

$$
\begin{aligned}
& \mathbf{R}_{X W_{1} X}=\mathbf{X}^{\mathbf{H}} \operatorname{diag}(\mathbf{s}) \mathbf{F} \operatorname{diag}\left(\frac{1}{\sqrt{\mathbf{E}_{\mathbf{x}}^{H} \mathbf{E}_{\mathbf{x}}}}\right) \mathbf{F}^{\mathbf{H}} \operatorname{diag}\left(\mathbf{s}^{\mathbf{H}}\right) \mathbf{X} \\
& \mathbf{R}_{X W_{2} X}=\mathbf{X}^{\mathbf{H}} \operatorname{diag}(\mathbf{s}) \mathbf{F} \operatorname{diag}\left(\frac{1}{\left|\mathbf{E}_{\mathbf{x}}\right|}\right) \mathbf{F}^{\mathbf{H}} \operatorname{diag}\left(\mathbf{s}^{\mathbf{H}}\right) \mathbf{X}
\end{aligned}
$$

Eq. 18 can now be easily used for maximization of the $\frac{l_{2}}{l_{1}}$-norm of the envelope spectrum. The generalized eigenvalue problem to be solved can be formulated as such:

$$
\mathbf{R}_{X W_{1} X} \mathbf{h}=\mathbf{R}_{X W_{2} X} \mathbf{h} \lambda
$$

The iterative algorithm used to maximize the $\frac{l_{2}}{l_{1}}$-norm consists of four basic steps:

1. Assume an initial guess for $\mathbf{h}$

2. Estimate $\mathbf{R}_{X W_{1} X}$ and $\mathbf{R}_{X W_{2} X}$ based on $\mathbf{h}$ and $\mathbf{X}$ using Eq. 20

3. Solve Eq. 21 to find $\lambda_{\max }$ and a new filter $\mathbf{h}$ that corresponds to a higher value of the used criterion

4. Return to step 2 using the new $\mathbf{h}$ until convergence is reached or the maximum number of iterations

These steps are used for all three indicator maximization procedures, specified in Sections 2.2.1, 2.2.2, and 2.2.3.

\subsubsection{Derivation of filter using Hoyer Index}

Since the Hoyer Index is essentially a normalized version of the $\frac{l_{2}}{l_{1}}$-norm, the derivation is very similar. The Hoyer index of the squared envelope spectrum $\mathbf{E}_{\mathbf{x}}$ is defined as:

$$
\text { Hoyer Index }=\left(\sqrt{N}-\frac{\sum_{n=1}^{N}\left|E_{x}(n)\right|}{\sqrt{\sum_{n=1}^{N}\left|E_{x}(n)\right|^{2}}}\right) \frac{1}{\sqrt{N}-1}
$$

Rewriting Eq. 22 using vector notation results in:

$$
\begin{aligned}
& \text { Hoyer Index }=\left(\sqrt{N}-\frac{\mathbf{E}_{\mathbf{x}}{ }^{H} \operatorname{diag}\left(\frac{1}{\mathbf{E}_{\mathbf{x}}} \mid\right) \mathbf{E}_{\mathbf{x}}}{\sqrt{\mathbf{E}_{\mathbf{x}}^{H} \mathbf{E}_{\mathbf{x}}}}\right) \frac{1}{\sqrt{N}-1} \\
& =\frac{\sqrt{N} \frac{\mathbf{E}_{\mathbf{x}}^{H} \mathbf{E}_{\mathbf{x}}}{\sqrt{\mathbf{E}_{x}^{H} \mathbf{E}_{\mathbf{x}}}-\mathbf{E}_{\mathbf{x}}^{H} \operatorname{diag}\left(\frac{1}{\left(\mathbf{E}_{\mathbf{x}}\right)}\right) \mathbf{E}_{\mathbf{x}}}}{(\sqrt{N}-1) \frac{\mathbf{E}_{\mathbf{x}} \mathbf{E}_{\mathbf{x}}}{\sqrt{\mathbf{E}_{\mathbf{x}}^{H} \mathbf{E}_{\mathbf{x}}}}} \\
& =\frac{\mathbf{E}_{\mathbf{x}}^{H} \operatorname{diag}\left(\frac{\sqrt{N}}{\sqrt{\mathbf{E}_{\mathbf{x}}^{H} \mathbf{E}_{\mathbf{x}}}}\right) \mathbf{E}_{\mathbf{x}}-\mathbf{E}_{\mathbf{x}}^{H} \operatorname{diag}\left(\frac{1}{\mid \mathbf{E}_{\mathbf{x}}}\right) \mathbf{E}_{\mathbf{x}}}{\mathbf{E}_{\mathbf{x}}^{H} \operatorname{diag}\left(\frac{\sqrt{N}-1}{\sqrt{\mathbf{E}_{\mathbf{x}}^{H} \mathbf{E}_{\mathbf{x}}}}\right) \mathbf{E}_{\mathbf{x}}} \\
& =\frac{\mathbf{E}_{\mathbf{x}}^{H} \operatorname{diag}\left(\frac{\sqrt{N}}{\sqrt{\mathbf{E}_{\mathbf{x}}^{H} \mathbf{E}_{\mathbf{x}}}}-\frac{1}{\left(\mathbf{E}_{\mathbf{x}}\right)}\right) \mathbf{E}_{\mathbf{x}}}{\mathbf{E}_{\mathbf{x}}^{H} \operatorname{diag}\left(\frac{\sqrt{N}-1}{\sqrt{\mathbf{E}_{\mathbf{x}}^{H} \mathbf{E}_{\mathbf{x}}}}\right) \mathbf{E}_{\mathbf{x}}}
\end{aligned}
$$

After some more rewriting, the generalized Rayleigh quotient emerges again: 


$$
\text { Hoyer Index }=\frac{\mathbf{h}^{\mathbf{H}} \mathbf{X}^{\mathbf{H}} \operatorname{diag}(\mathbf{s}) \mathbf{F} \operatorname{diag}\left(\frac{\sqrt{N}}{\sqrt{\mathbf{E}_{x}^{\mathbf{H}} \mathbf{E}_{\mathbf{x}}}}-\frac{1}{\mid \mathbf{E}_{\mathbf{x}}}\right) \mathbf{F}^{\mathbf{H}} \operatorname{diag}\left(\mathbf{s}^{\mathbf{H}}\right) \mathbf{X h}}{\mathbf{h}^{\mathbf{H}} \mathbf{X}^{\mathbf{H}} \operatorname{diag}(\mathbf{s}) \mathbf{F} \operatorname{diag}\left(\frac{\sqrt{N}-1}{\sqrt{\mathbf{E}_{x}^{\mathbf{E}} \mathbf{E}_{\mathbf{x}}}}\right) \mathbf{F}^{\mathbf{H}} \operatorname{diag}\left(\mathbf{s}^{\mathbf{H}}\right) \mathbf{X h}}=\frac{\mathbf{h}^{\mathbf{H}} \mathbf{R}_{X W_{1} X} \mathbf{h}}{\mathbf{h}^{\mathbf{H}} \mathbf{R}_{X W_{2} X} \mathbf{h}}
$$

\subsubsection{Derivation of filter using spectral negentropy}

The spectral negentropy $\Delta I_{E}$ is the negative of spectral entropy and is defined for the envelope spectrum as:

$$
\Delta I_{E}=-H_{E}=\left\langle\frac{\left|E_{x}(\alpha)\right|^{2}}{\left\langle\left|E_{x}(\alpha)\right|^{2}\right\rangle} \ln \left(\frac{\left|E_{x}(\alpha)\right|^{2}}{\left\langle\left|E_{x}(\alpha)\right|^{2}\right\rangle}\right)\right\rangle
$$

Rewriting again using vector notation:

$$
\begin{aligned}
& \Delta I_{E}=\frac{N}{\mathbf{E}_{\mathbf{x}}^{H} \mathbf{E}_{\mathbf{x}}}\left\langle\left|E_{x}(\alpha)\right|^{2} \ln \left(\frac{\left|E_{x}(\alpha)\right|^{2}}{\left\langle\left|E_{x}(\alpha)\right|^{2}\right\rangle}\right)\right\rangle \\
& =\frac{\mathbf{E}_{\mathbf{x}}^{H} \operatorname{diag}\left(\ln \left(\frac{\left|\mathbf{E}_{\mathbf{x}}\right|^{2}}{\left\langle\left\langle\left.\mathbf{E}_{\mathbf{x}}\right|^{2}\right\rangle\right.}\right)\right) \mathbf{E}_{\mathbf{x}}}{\mathbf{E}_{\mathbf{x}}^{H} \mathbf{E}_{\mathbf{x}}}
\end{aligned}
$$

This can again be written as a generalized Rayleigh quotient.

$$
\Delta I_{E}=\frac{\mathbf{h}^{\mathbf{H}} \mathbf{X}^{\mathbf{H}} \operatorname{diag}(\mathbf{s}) \mathbf{F} \operatorname{diag}\left(\ln \left(\frac{\left|\mathbf{E}_{\mathbf{X}}\right|^{2}}{\left\langle\left|\mathbf{E}_{\mathbf{X}}\right|^{2}\right\rangle}\right)\right) \mathbf{F}^{\mathbf{H}} \operatorname{diag}\left(\mathbf{s}^{\mathbf{H}}\right) \mathbf{X h}}{\mathbf{h}^{\mathbf{H}} \mathbf{X}^{\mathbf{H}} \operatorname{diag}(\mathbf{s}) \mathbf{F} \mathbf{F}^{\mathbf{H}} \operatorname{diag}\left(\mathbf{s}^{\mathbf{H}}\right) \mathbf{X h}}=\frac{\mathbf{h}^{\mathbf{H}} \mathbf{R}_{X W_{1 E} X} \mathbf{h}}{\mathbf{h}^{\mathbf{H}} \mathbf{R}_{X W_{2 E} X} \mathbf{h}}
$$

The spectral negentropy also has the useful property that it is always greater than zero and smaller than $\ln (N)$ :

$$
0 \leqslant \Delta I_{E} \leqslant \ln (N)
$$

The same is valid for the negentropy in the time domain $\Delta I_{\epsilon}$. This is proven in Appendix A A.

\subsection{Practical considerations}

The maximization of the sparsity of the envelope spectrum is based on the assumption that the main component increasing the sparsity is the potential fault signature. Therefore, the signal first needs to be whitened such that the deterministic components are removed before solving the maximization problem since these components often introduce high amplitude discrete peaks in the envelope spectrum. This whitening or removal of deterministic components can be achieved with various techniques, e.g. time-synchronous averaging [46], cepstrum editing [47,48], discrete/random separation [49], selfadaptive noise cancellation [50], etc.

The calculation of correlation matrices $\mathbf{R}_{X W_{1} X}$ and $\mathbf{R}_{X W_{2} X}$ includes using a Fourier matrix to calculate the envelope spectrum. However, in the actual calculation this can be replaced by an FFT to improve the computation time and the memory requirements. The total duration of the computations will depend mainly on the signal length and the chosen filter length. Since the matrices can become quite large for long signals and filters, the number of multiplications and FFTs that need to be calculated can lead to long computation times.

\subsection{Extension for non-stationary angular speed cases}

A potential extension of the method is to make the kernel of the Fourier matrix used in the filter correlation matrices dependent on angle instead of time. This way the instantaneous angular speed variations can be taken into account directly in the filter. This extension is not further investigated in this paper but can be easily achieved by means of the Velocity Synchronous Discrete Fourier Transform (VSDFT) [51]. The Fourier matrix then becomes:

$$
\mathbf{F}=\frac{1}{\Theta}\left[\begin{array}{cccccc}
\dot{\theta}_{0} & \dot{\theta}_{0} & \ldots & \dot{\theta}_{0} & \ldots & \dot{\theta}_{0} \\
\dot{\theta}_{1} & \dot{\theta}_{1} e^{-j \theta_{1} \Omega_{1}} & \ldots & \dot{\theta}_{1} e^{-j \theta_{1} \Omega_{k}} & \ldots & \dot{\theta}_{1} e^{-j \theta_{1} \Omega_{K-1}} \\
\vdots & \vdots & \ddots & & & \vdots \\
\dot{\theta}_{n} & \dot{\theta}_{n} e^{-j \theta_{n} \Omega_{1}} & \ldots & \dot{\theta}_{n} e^{-j \theta_{n} \Omega_{k}} & \ldots & \dot{\theta}_{n} e^{-j \theta_{n} \Omega_{K-1}} \\
\vdots & \vdots & & & \ddots & \vdots \\
\dot{\theta}_{L-N-1} & \dot{\theta}_{L-N-1} e^{-j \theta_{L-N-1} \Omega_{1}} & \ldots & \dot{\theta}_{L-N-1} e^{-j \theta_{L-N-1} \Omega_{k}} & \ldots & \dot{\theta}_{L-N-1} e^{-j \theta_{L-N-1} \Omega_{K-1}}
\end{array}\right]
$$

with $\theta_{n}=\Sigma_{n=1}^{L} \dot{\theta}(n), \Omega(k)$ the vector of orders for the representation of the order spectrum, and the normalization factor $\Theta=\Sigma_{n=1}^{L} \dot{\theta}(n)$.

An alternative approach would be to resample the signal to the angular domain before passing it to the filter optimization. 


\subsection{Extension for single-input multiple-output (SIMO) systems}

Similar to the description in [35], the indicator calculations described here can also be extended for the case when there is more than one acquisition channel that measures the vibration response. The proposed approach allows a fairly straightforward extension to the case where one has Q responses $\mathbf{x}_{q}$. Each response of $\mathbf{x}_{q}$ filtered by $\mathbf{h}_{q}$ can be summed together then to return an estimation of $\mathbf{s}$. The iterative procedure using the Rayleigh quotient can still be used for the SIMO case. The only adaptation necessary is of the correlation matrices and the filter vector.

$\mathbf{R}_{X W_{1} X}$ and $\mathbf{R}_{X W_{2} X}$ need to be expressed as cross-correlation matrices:

$$
\mathbf{R}_{X W X}=\left[\begin{array}{ccc}
\ddots & & \mathbf{R}_{q W \tilde{q}} \\
& \mathbf{R}_{q W q} & \\
\mathbf{R}_{\tilde{q} W q} & & \ddots
\end{array}\right]
$$

with $\mathbf{R}_{q W q}$ the weighted auto-correlation matrix of $\mathbf{x}_{q}$ and the off-diagonal matrices are the weighted cross-correlation matrices of $\mathbf{x}_{q}$ and $\mathbf{x}_{\tilde{q}}$. The filter $\mathbf{h}$ then becomes:

$$
\mathbf{h}=\left[\begin{array}{c}
\mathbf{h}_{1} \\
\vdots \\
\mathbf{h}_{q} \\
\vdots \\
\mathbf{h}_{Q}
\end{array}\right]
$$

The $q^{\text {th }}$ contribution to the signal of interest $\mathbf{s}$ is then calculated as:

$$
\mathbf{s}_{q}=\Sigma_{q=1}^{Q} \mathbf{X}_{q} \mathbf{h}_{q}
$$

with $\mathbf{X}_{q}$ the Toeplitz matrix of $\mathbf{x}_{q}$ as defined in Eq. 6. Thus, the overall signal of interest $\mathbf{s}$ is then found by:

$$
\mathbf{s}=\Sigma_{q=1}^{Q} \mathbf{s}_{q}
$$

\section{Simulation analysis}

To validate and illustrate the proposed approach, two simulated cases are first considered. To add some point of reference, the performance of the proposed approach with the three different sparsity indicators is compared to time-domain blind deconvolution filtering based on Minimum Entropy Deconvolution (MED), ICS2, and the time-domain spectral negentropy. Two cases with cyclostationary source signals are examined:

- Case 1: Periodic impulses with Gaussian distributed amplitudes

- Case 2: Periodic impulses with Gaussian distributed amplitudes and randomly spaced impulses representing the slip in bearings

These two cases are the same as the first two cases in [35] to provide additional reference for comparison. The periodic impulse signal $s_{1}$ is convolved with the IRF $g_{1, s}$ and the Gaussian noise with $g_{1, n}$. The generated signals are shown separately in Fig. 2. Instead of only analyzing a single simulation though, the additive Gaussian noise is varied in signal-to-noise ratio (SNR) from $-80 \mathrm{~dB}$ to $0 \mathrm{~dB}$ to assess the trending behavior of the filters. The overall variance of the full signal is kept constant. For the time-domain blind filters, a filter length of 40 samples is used, while the sparsity-based spectral filters use a filter length of 20 samples. The maximum number of iterations is set to 50 .

Fig. 3 shows the resulting indicator evolutions from $-80 \mathrm{~dB}$ to $0 \mathrm{~dB}$ SNR for the first case. It can be seen that in this particular case the ICS2 filter outperforms MED and time-domain negentropy filtering. The filters based on the sparsity indicators show a significant increase at approximately $-38 \mathrm{~dB}$, similar to the ICS2 filter.

To inspect the results further, Fig. 4 displays a color map of the squared envelope spectra for the first case. The envelope spectra of the time-domain filters (ICS2, MED, and time-domain negentropy) exhibit clear harmonics of the fault frequency at $4 \mathrm{~Hz}$ for higher SNRs. In contrast, the envelope spectra of the sparsity-based filters exhibit the first fault harmonic at $4 \mathrm{~Hz}$ but also a lower frequency component that is higher in amplitude. This is due to the envelope spectrum of the simulation signal containing a very low frequency modulation due to the employed Gaussian distributed amplitudes of the periodic impulses. Additionally, this low frequency modulation increases the sparsity of the envelope spectrum.

Lastly, the filtered signals of case 1 are shown in Fig. 5 at the SNR of $-19 \mathrm{~dB}$, similar to the comparison of signals shown in [35]. The amplitude of the signals has been normalized between -1 and +1 . It turns out that the time-domain filters deconvolve the periodic impulse train of the input source signal, while the envelope spectrum sparsity-based filters extract the 

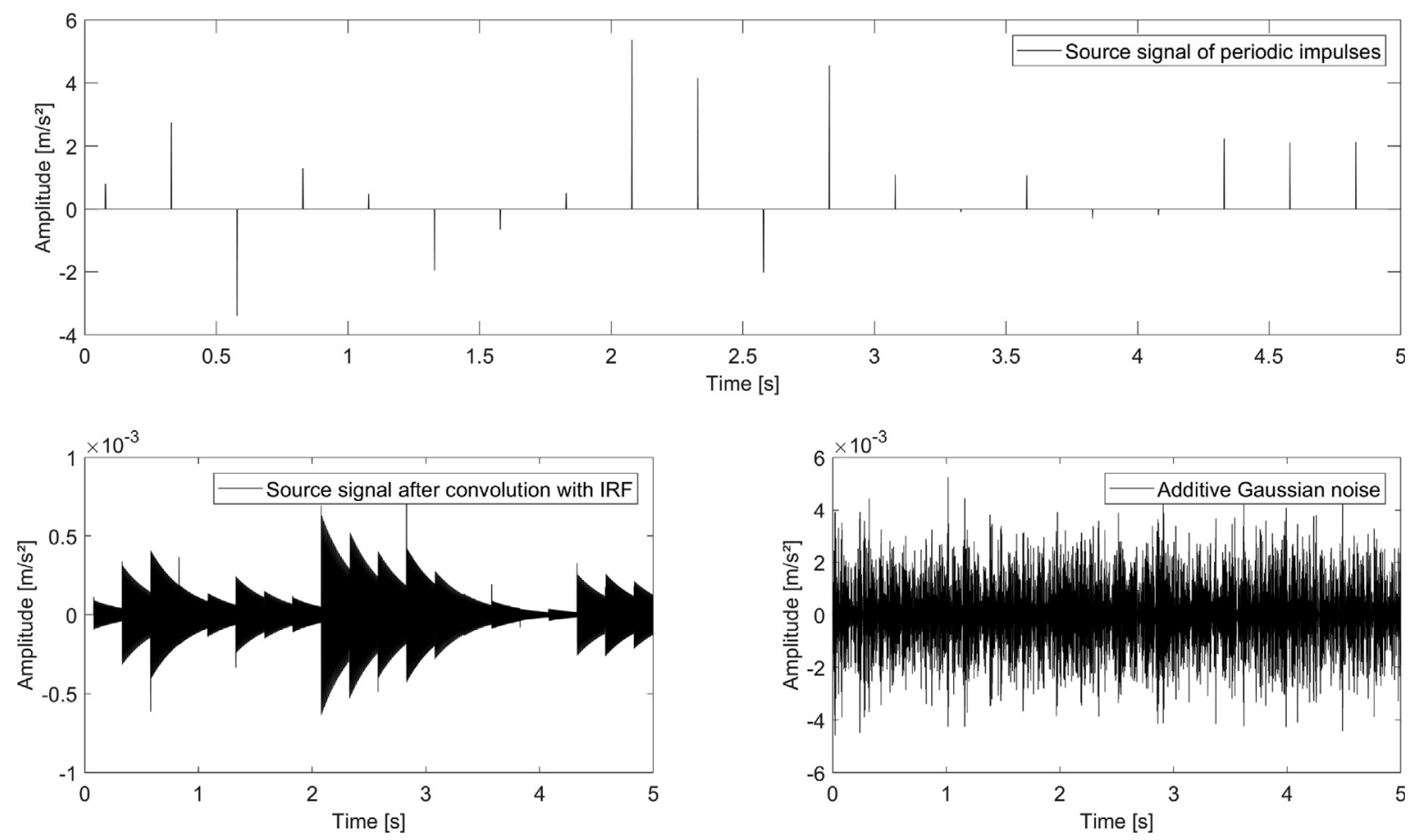

Fig. 2. Input signals used for simulation case 1: (Top) Periodic impulses with Gaussian distributed amplitudes $s_{1}$, (Bottom-left) after convolution with IRF $g_{1, s}$, (Bottom-right) Additive Gaussian noise after convolution with IRF $g_{1, n}$.
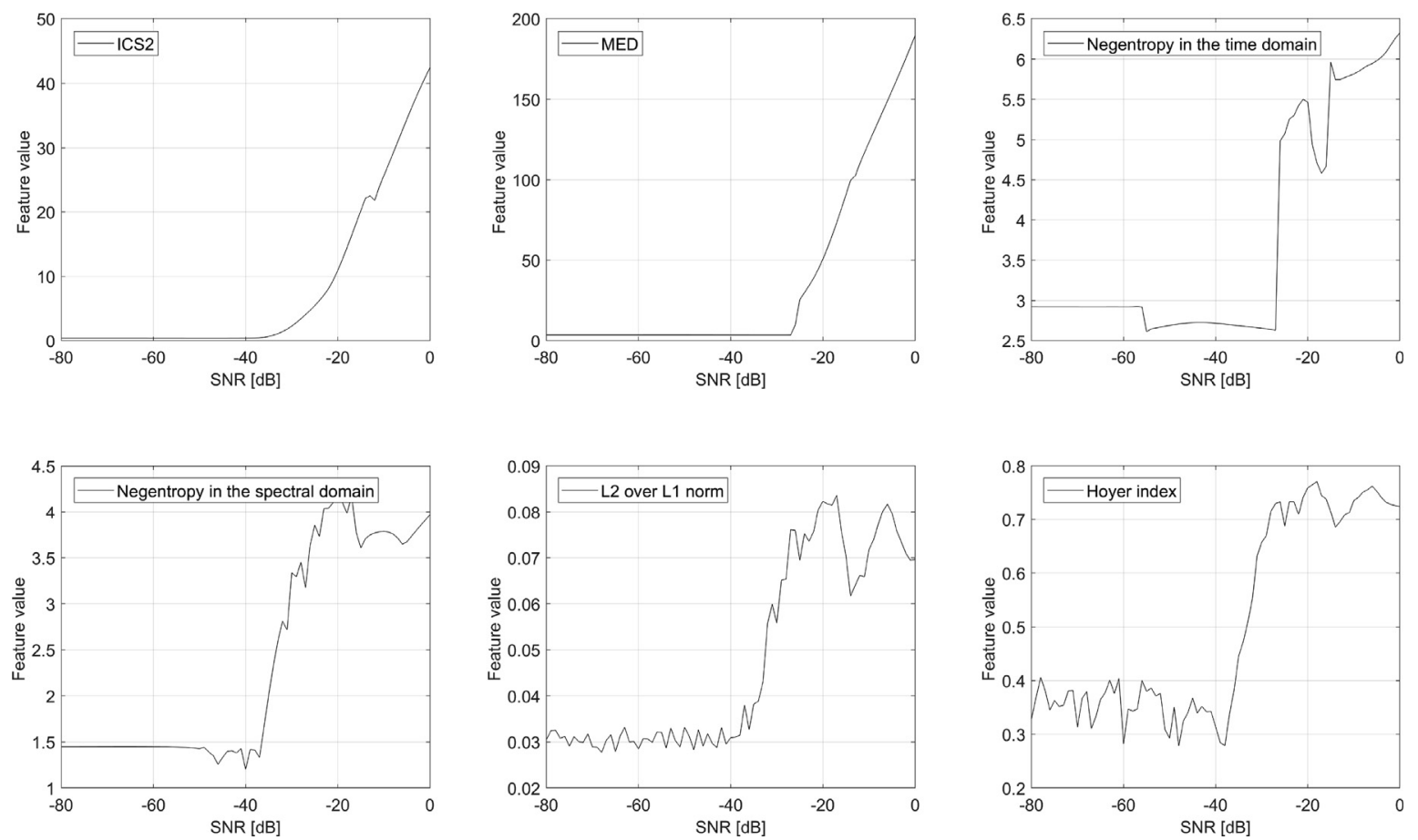

Fig. 3. Trending of the different indicators for simulation case 1.

periodic impulse train after deconvolution with its IRF. This result makes sense considering that the most sparse envelope spectrum is that of a signal with a pure sinusoidal amplitude modulation. A Dirac impulse train however results in an envelope spectrum that exhibits multiple harmonics which decreases the sparsity as compared to a single discrete peak. Therefore 

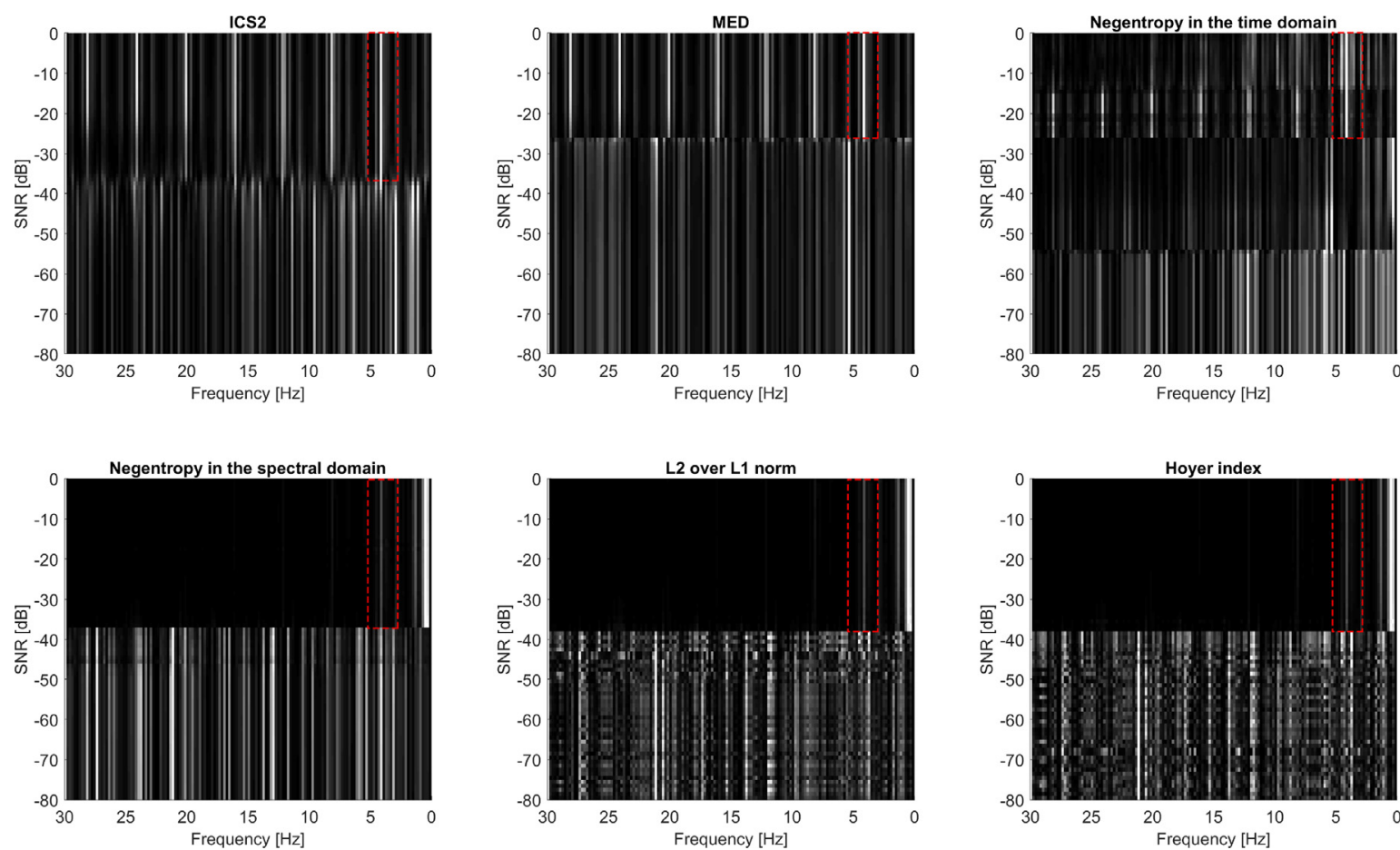

Fig. 4. Trending of the normalized squared envelope spectra for simulation case 1 . The fault frequency of interest is $4 \mathrm{~Hz}$. $($ Black $=0$, white $=1$ )
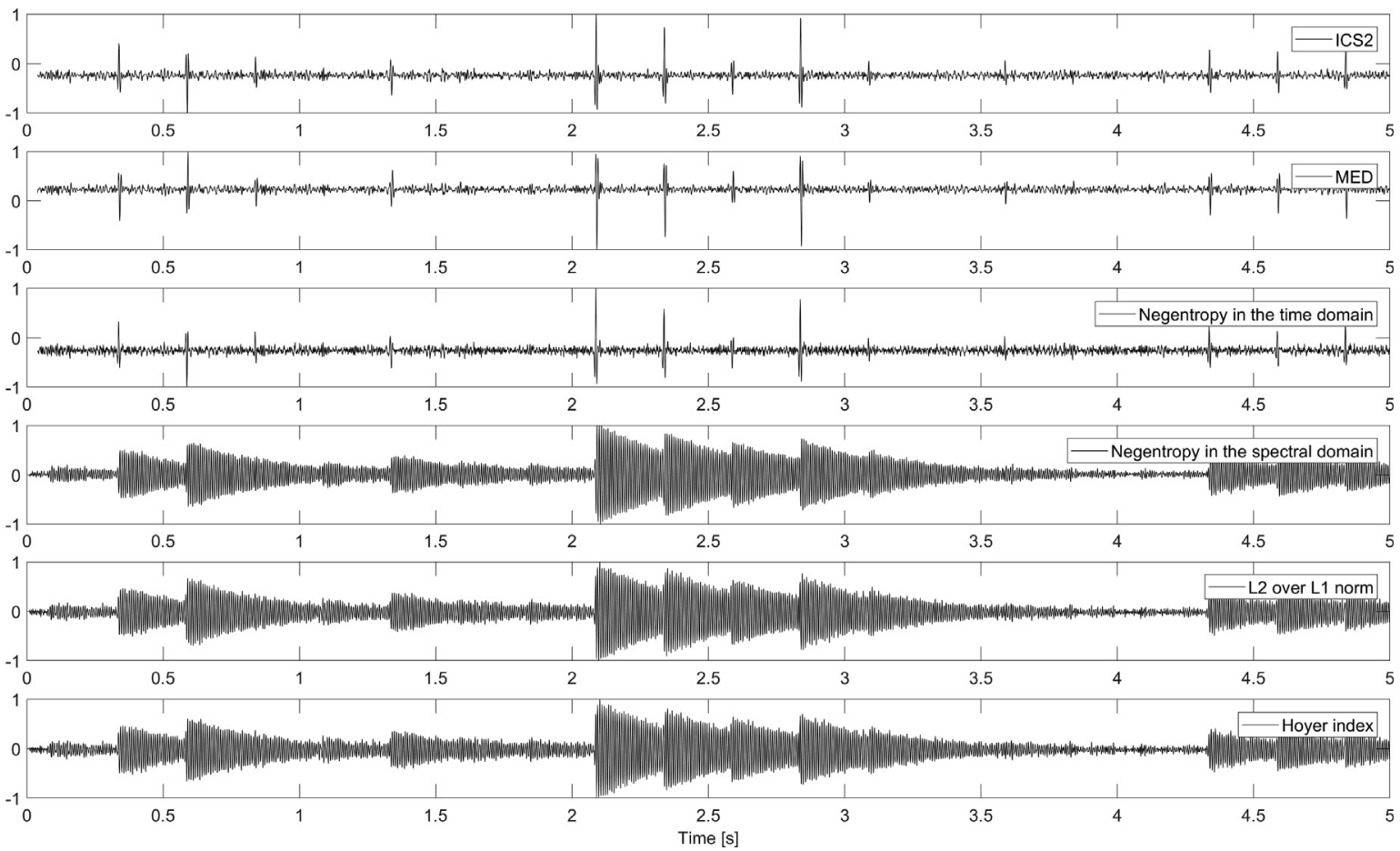

Fig. 5. Time waveforms at $-19 \mathrm{~dB}$ SNR for simulation case 1 . The amplitudes have been normalized between -1 and +1 .

the sparsity-based methods filter out the impulse train after convolution with the IRF which leads to a sparsity that is inbetween that of a pure sinusoidal modulation and that of an impulse train. For the purpose of condition monitoring, the non-removal of the convolution with the IRF is not essential in the sense that fault detection through tracking the fault 
frequency peak in the envelope spectrum remains the same. The signature of the envelope harmonics will differ though between the situation with and without convolution with the IRF. Also the evolution of the fault can be tracked through the envelope spectrum sparsity regardless of the IRF convolution since it is assumed that the main second-order cyclostationary component originates from the fault source.

The second example investigates the case where the fundamental fault period of the impulses fluctuate due to slip. The results are similar to the first case since the slip only impacts the filtering potential of the sparsity-based filters slightly. Figs. 6-8 display respectively the indicator trends, the envelope spectra color maps, and the filtered time waveforms at $-19 \mathrm{~dB}$. The sparsity values are only marginally lower than the ones for the first case without slip. This can be explained since the slip of the fault period causes the discrete envelope spectrum peaks to spread out over multiple frequency bins and thus reduce the sparsity of the envelope spectrum.

\section{Experimental analysis \& results}

The proposed approach is applied on vibration signals from the Prognostics Data Repository of NASA as provided by the Center for Intelligent Maintenance Systems (IMS) of the University of Cincinnati [52]. This data set was measured during a run-to-failure experiment in which a bearing experienced an outer race fault. The bearing test rig, as shown in Fig. 9, consists of a shaft coupled to an AC motor rotating at 2000 RPM. A radial load of $2700 \mathrm{~kg}$ is applied. Four Rexnord ZA-2115 bearings are mounted on the shaft together with high-end accelerometers. In total three run-to-failure experiments were conducted where one second measurements were recorded at a sample rate of $20 \mathrm{kHz}$ every $10 \mathrm{~min}$. The approach described in this paper is examined on the second data set containing 984 measurement samples and in which Bearing 1 failed due to an outer race defect.

Again, all six filters used in the simulation analysis are employed here to provide some reference for comparison of the methods' performance. The characteristic fault frequency of the outer race is approximately $236 \mathrm{~Hz}$ and is thus used as input for the ICS2 filter. The filter length is set at 20 samples and the maximum number of iterations is 50 . Theoretically the ICS2 filter should be most capable in tracking signal signature changes due to the fault emergence since it tracks the fault frequency modulation directly.

The results shown in Fig. 10 seem to confirm this theory. The ICS2 filter manages to already show a rise in ICS2 indicator at the start of day 4. The MED filter and the time-domain negentropy filter perform badly which comes as no surprise since it was already proven in [54] that kurtosis or other indicators tracking non-Gaussianity perform badly for this particular data set. The signal is in fact already non-Gaussian from the start of the test. These two filters therefore underperform in effectively tracking the fault in this case. The envelope spectrum sparsity-based filters on the other hand suggest the increase of mainly cyclostationarity of the signal due to the fault. All three filters exhibit a sudden increase in indicator value around the
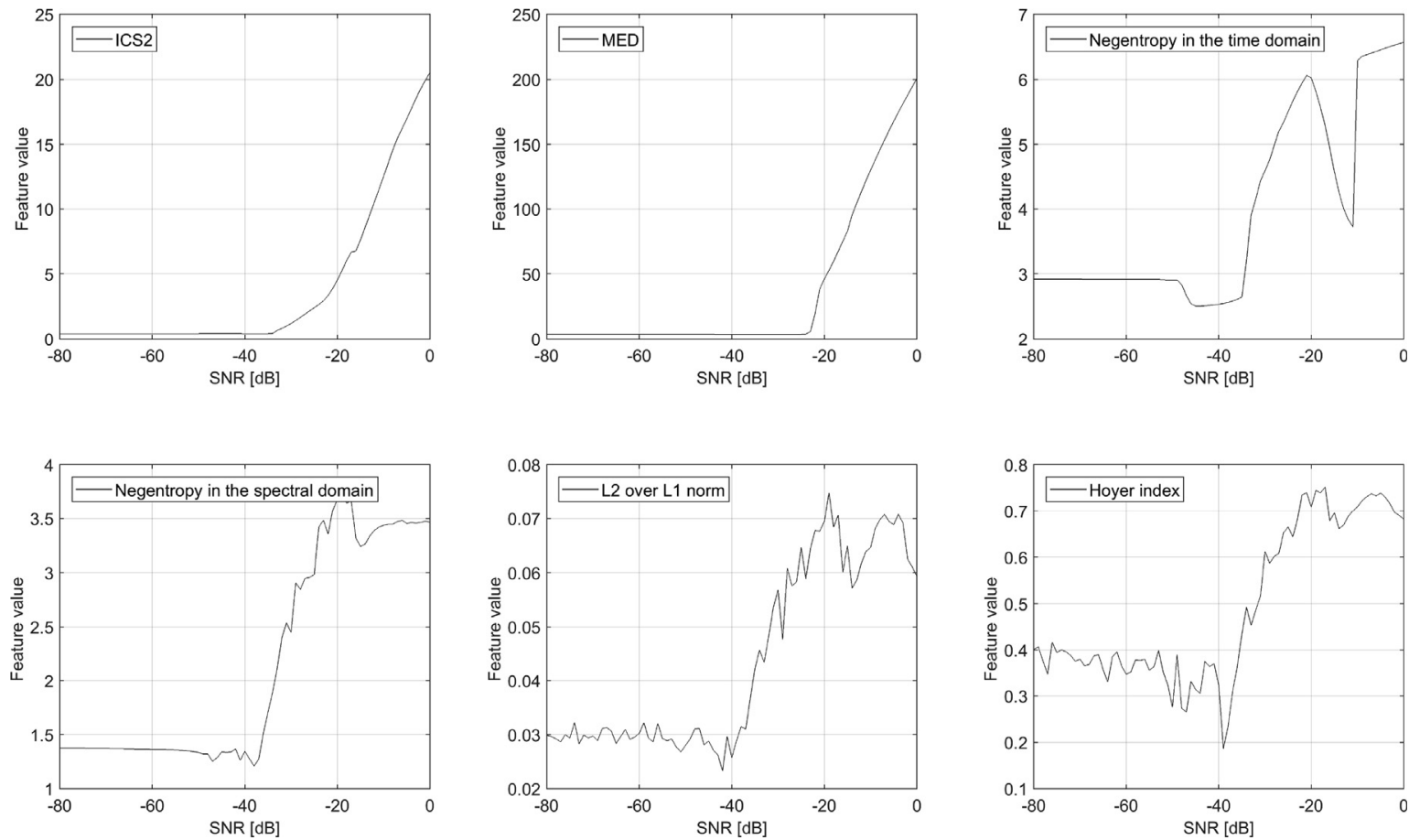

Fig. 6. Trending of the different indicators for simulation case 2 . 

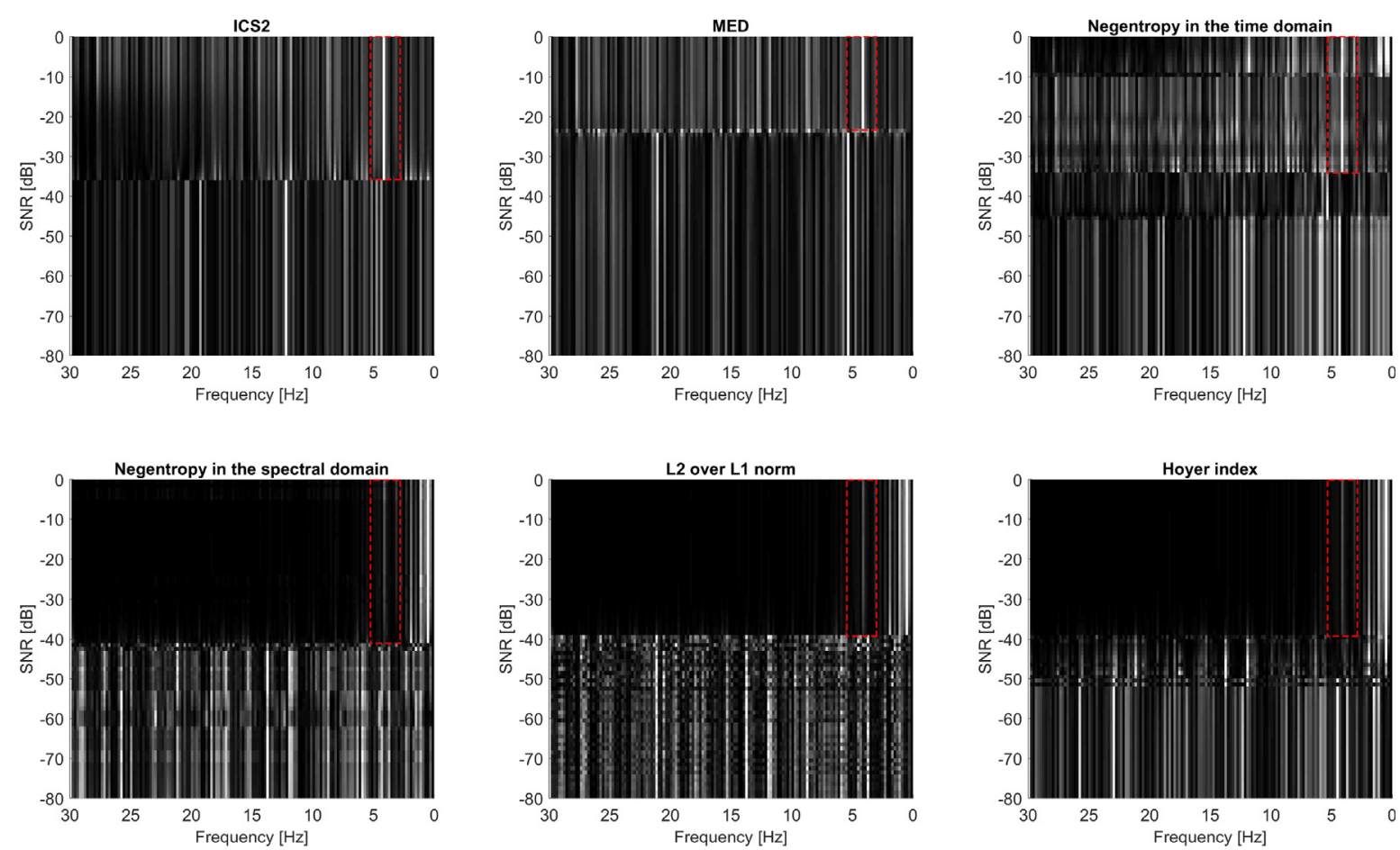

Fig. 7. Trending of the squared envelope spectra for simulation case 2 . The fault frequency of interest is $4 \mathrm{~Hz}$. (Black $=0$, white $=1$ ).

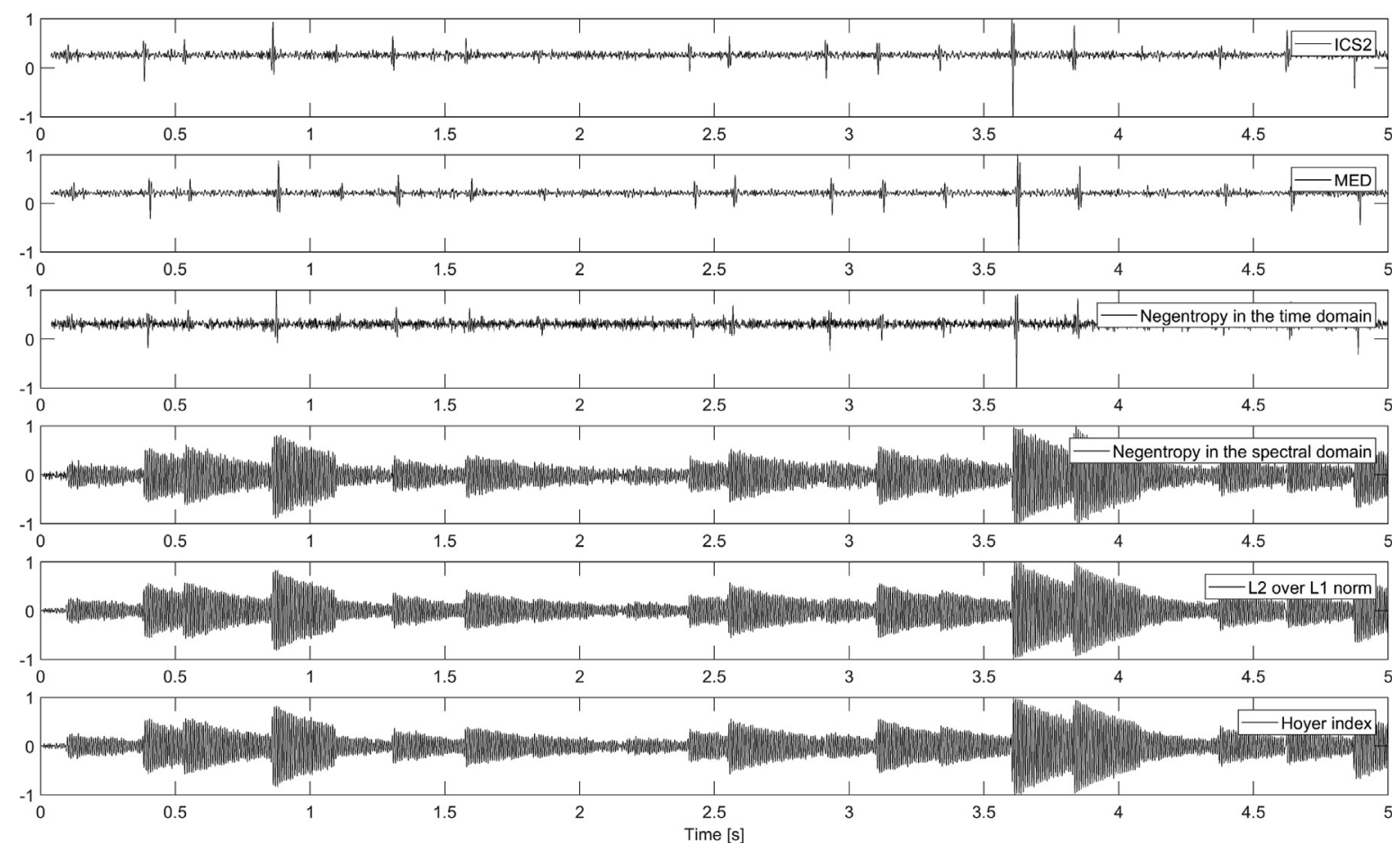

Fig. 8. Time waveforms at $-19 \mathrm{~dB}$ SNR for simulation case 2 . The amplitudes have been normalized between -1 and +1 . 


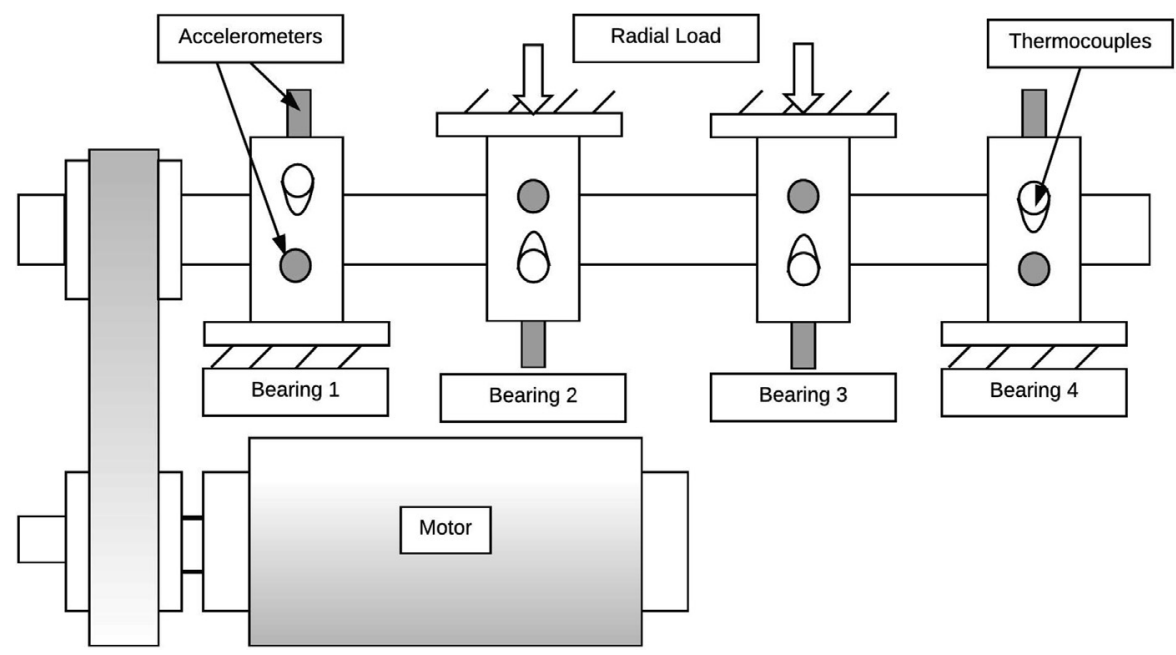

Fig. 9. Bearing test rig [53] of the IMS dataset.
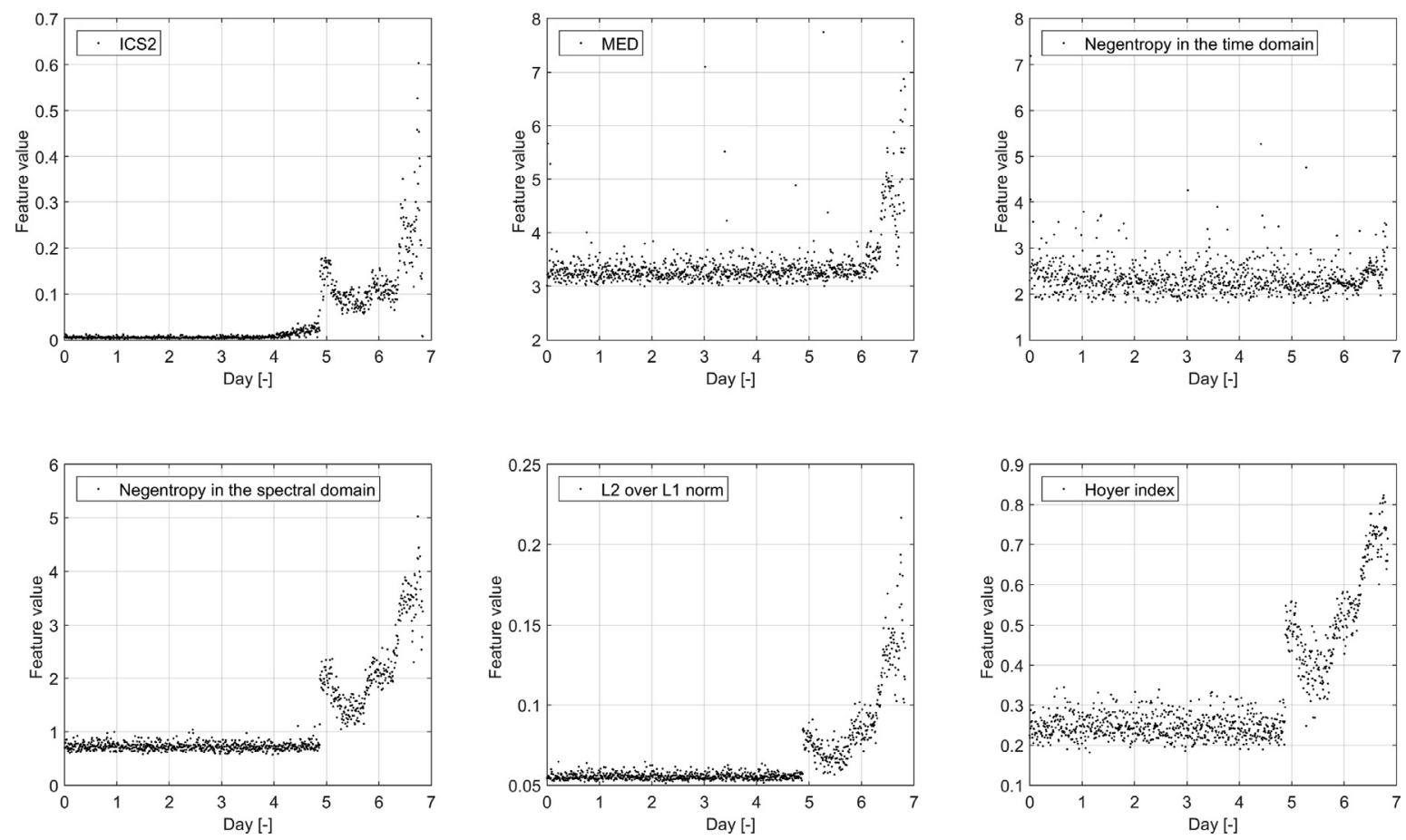

Fig. 10. Evolution of the indicators after filtering with the six different methods on the IMS data set. (Top row, left to right) ICS2 filter, MED filter, Negentropy in the time-domain filter. (Bottom row, left to right) Filters based on respectively the negentropy, the $\frac{l_{2}}{l_{1}}$-norm, and Hoyer Index of the envelope spectrum.

start of day 5. As described in [54], the degradation of the bearing mainly manifests itself by a significant surge in cyclostationarity and not non-Gaussianity.

The increase in envelope spectrum sparsity means that a dominant discrete peak or set of discrete peaks should show up in the envelope spectrum. Fig. 11 shows a waterfall colormap plot of the envelope spectra as a function of time from $0 \mathrm{~Hz}$ to $1 \mathrm{kHz}$. The fault frequency at $236 \mathrm{~Hz}$ can be detected clearly starting from day 4 for the ICS2 filter, and from day 5 for the sparsity-based filters. The filtering singles out the fault frequency in the envelope spectrum from the noise as showcased by the difference in envelope spectrum before and after filtering shown in Fig. 12. This makes inspection of the signal for the type of fault significantly easier and quicker. 

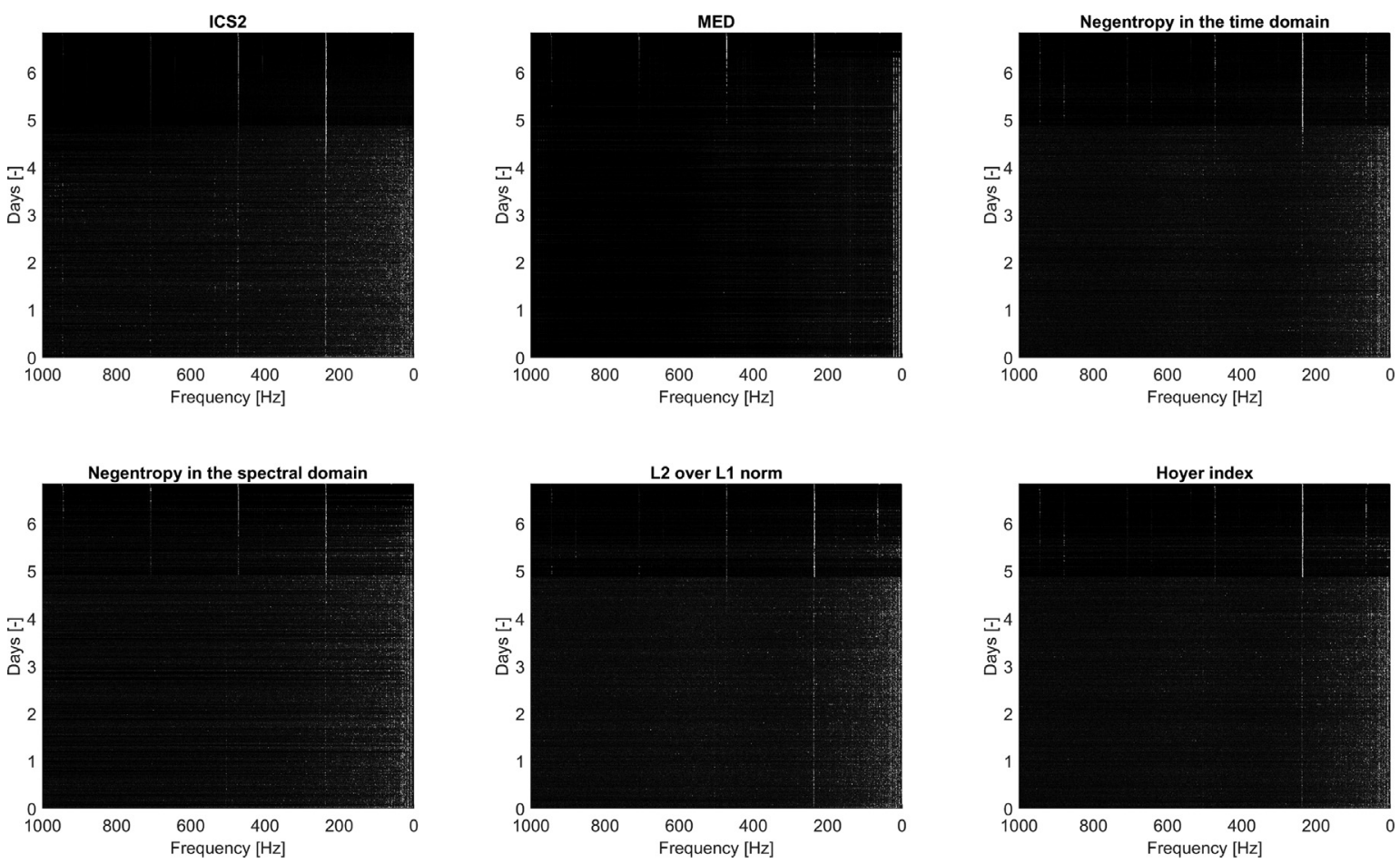

Fig. 11. Waterfall plots of the normalized envelope spectra after filtering with the six different methods on the IMS data set. (Top row, left to right) ICS2 filter, MED filter, Negentropy in the time-domain filter. (Bottom row, left to right) Filters based on respectively the negentropy, the $\frac{l_{2}}{l_{1}}$-norm, and Hoyer Index of the envelope spectrum. (Black $=0$, white $=1$ ).
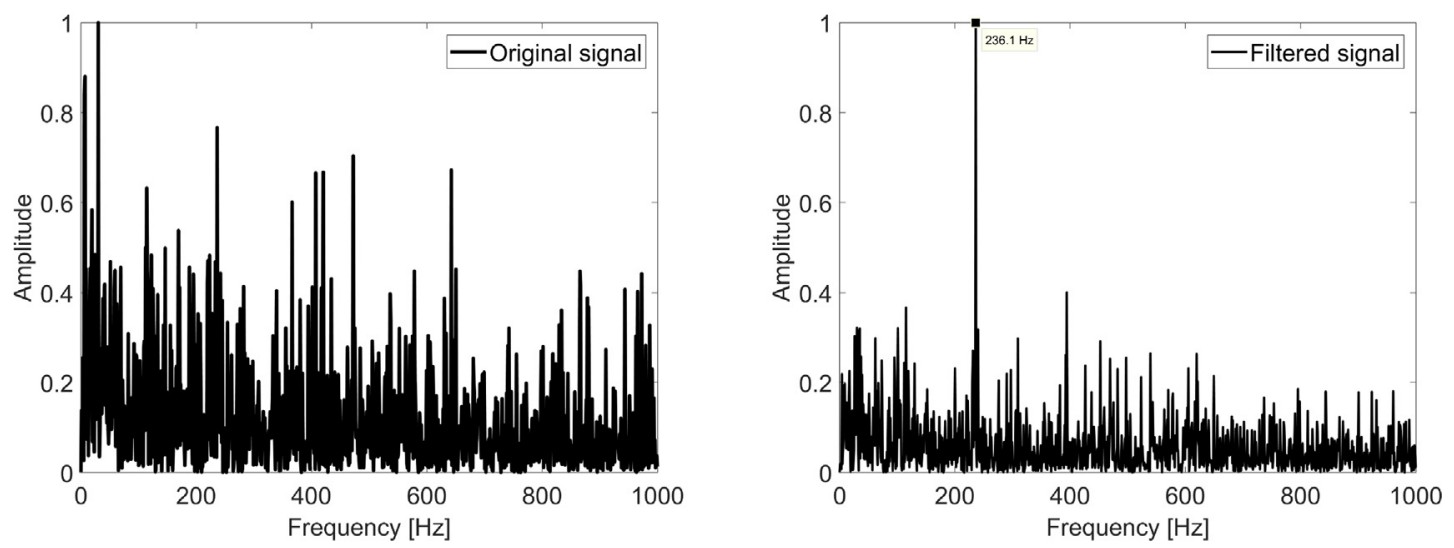

Fig. 12. Envelope spectrum after filtering with the Hoyer Index filter for measurement ndeg690.

\section{Discussion}

The simulation and experimental results showcase the potential for condition monitoring using a blind filtering approach of vibration signals without any prior knowledge of the fault frequencies. The simulation results indicate that filtering based on the sparsity of the envelope spectrum can outperform traditional time indicator-based filtering approaches such as MED. This is especially so when the fault signature introduces cyclostationarity in the signal which is detected effectively by the envelope spectrum. A limitation of kurtosis-based filtering is exemplified for the case when the fault impulses have a high repetition rate and do not fully decay before the next impulse. In this case the kurtosis will only be affected slightly due to the lack of strong non-Gaussian behavior. This phenomenon can occur when dealing with very fast rotating machines where the bearing frequencies are in the $\mathrm{kHz}$-range. 
Another significant difference in the way the sparsity-based filters function as compared to more conventional blind deconvolution filters such as MED is the fact that the proposed filters purely try to maximize a certain property of the signal. This means that they do not recover source signals (e.g. repetitive impacts), separate signals from a mixture, or denoise the signal. This is a different blind filtering perspective than typically used in the past. As a consequence, the user needs to keep this property in mind when analyzing the results since the filtered response might not accurately represent the signal of interest, rather a distorted version of it that maximizes the used criterion. Nevertheless, the experimental applicability of the suggested approach is fairly apparent since the envelope spectrum is one of the most prevalent tools in vibrationbased condition monitoring.

The experimental application illustrates the sensitivity of the proposed approach to cyclostationary sources. While the sparsity-based filters is slightly slower in detecting the fault than the ICS2 filter, they still manage to track the fault accurately without any knowledge of the actual bearing fault frequency. The results suggest as well that simply looking at the envelope spectrum sparsity of the filtered signal can be a reliable blind tracking measure for the amount of cyclostationarity in a signal. Lastly, the results do not give any strong indication for one of the three proposed sparsity measures to be superior in terms of tracking capability.

The proposed approach employs a powerful new way of looking at the combination of blind filtering and the envelope spectrum. As is the case for almost every method however, there are some drawbacks or hindrances that can negatively influence results. The main difficulty in using this type of filters resides in the choice for the filter length. This length can impact the results and the computation time significantly. Obviously longer filter lengths increase the computation time, but they can also lead to slower convergence or non-convergence of the filter coefficients. The latter can be especially troublesome if the indicator values oscillate strongly due to this non-convergence. Therefore it is recommended to keep the filter length short, while maintaining the capability of the filter in suppressing unwanted frequency content.

Despite this obstacle, this paper hopes to pave the way for further research in novel blind filtering approaches for vibration monitoring. The Rayleigh quotient iteration framework detailed in this paper is the main tool responsible for the ease of implementation of the proposed filters. Yet this does not mean other approaches are not viable. A future improvement could be to reduce the sensitivity to the filter length and improve the convergence behavior.

\section{Conclusion}

This paper investigates novel blind filtering techniques that utilize the sparsity of the envelope spectrum to track the presence of faults with a second-order cyclostationary signature in vibration signals. Three sparsity measures are used to derive filter formulations that are applicable in a methodology based on Rayleigh quotient iteration. This approach allows for fast computation and straightforward implementation of the proposed filters.

Both the simulation and experimental results indicate the efficacy of the proposed blind filters in tracking the emergence of cyclostationary sources. The three suggested sparsity measures perform in a similar manner with regards to their rise in absolute values and to their corresponding filtering behavior. When the fault signal does not exhibit strong impulsive content, the described approach can outperform conventional blind deconvolution methods such as MED and its variants. Blind filters that do employ prior knowledge of the fault frequencies such as the ICS2 filter do still outperform the proposed filter methodology, which is not surprising considering the narrower frequency range of interest.

Future work will focus on improving the stability of the results so that filter length plays less of an influential role in the computation time and convergence behavior of the algorithm.

\section{Declaration of Competing Interest}

The authors declare that they have no known competing financial interests or personal relationships that could have appeared to influence the work reported in this paper.

\section{Acknowledgment}

The authors of VUB would like to thank Fonds Wetenschappelijk Onderzoek (FWO) for their support with an SB Ph.D. fellow grant and the agency for Innovation by Science and Technology in Belgium for supporting the Strategisch Basis Onderzoek Hypermodeling strategies for operational optimization(SBO HYMOP) project and the Fulbright foundation for facilitating collaboration with MIT. The authors of VUB would also like to thank Strategic Initiative Materials in Flanders (SIM) for the Material and Signal-Processing based prediction of WEC probability (SBO MASIWEC) project. They would also like to thank FWO for providing funding for a long stay abroad of Cédric Peeters at the university of INSA Lyon.

\section{Appendix A. Appendix A}

It can be proven that the spectral negentropy is always greater than zero and smaller than $\ln (N)$ :

$$
0 \leqslant \Delta I_{E} \leqslant \ln (N)
$$


Starting from the Shannon (or information) entropy $H$, the following is valid:

$$
H=-\sum_{i} p_{i} \ln \left(p_{i}\right) \geqslant 0 \text { and } \sum_{i} p_{i}=1
$$

with $p_{i}$ a discrete set of probabilities. Thus for $p_{i}=\frac{\left|\mathbf{E}_{\mathbf{i}}\right|^{2}}{\sum_{i}^{N}\left|\mathbf{E}_{\mathbf{i}}\right|^{2}}$ the following is true:

$$
\begin{aligned}
& \sum_{i}^{N} \frac{\left|\mathbf{E}_{\mathbf{i}}\right|^{2}}{\sum_{i}^{N}\left|\mathbf{E}_{\mathbf{i}}\right|^{2}} \ln \left(\frac{\left|\mathbf{E}_{\mathbf{i}}\right|^{2}}{\sum_{i}^{N}\left|\mathbf{E}_{\mathbf{i}}\right|^{2}}\right) \leq 0 \\
& \left.\frac{1}{N} \sum_{i}^{N} \frac{\left|\mathbf{E}_{\mathbf{i}}\right|^{2}}{\frac{1}{N} \sum_{i}^{N}\left|\mathbf{E}_{\mathbf{i}}\right|^{2}} \ln \frac{\frac{1}{N}\left|\mathbf{E}_{\mathbf{i}}\right|^{2}}{\frac{1}{N} \sum_{i}\left|\mathbf{E}_{\mathbf{i}}\right|^{2}}\right) \leq 0 \\
& \left\langle\frac{\left|\mathbf{E}_{\mathbf{i}}\right|^{2}}{\left\langle\left|\mathbf{E}_{\mathbf{i}}\right|^{2}\right\rangle} \ln \left(\frac{\left|\mathbf{E}_{\mathbf{i}}\right|^{2}}{\left\langle\left|\mathbf{E}_{\mathbf{i}}\right|^{2}\right\rangle}\right)+\frac{\left|\mathbf{E}_{\mathbf{i}}\right|^{2}}{\left\langle\left|\mathbf{E}_{\mathbf{i}}\right|^{2}\right\rangle} \ln \left(\frac{1}{N}\right)\right\rangle \leq 0 \\
& \left\langle\frac{\left|\mathbf{E}_{\mathbf{i}}\right|^{2}}{\left\langle\left|\mathbf{E}_{\mathbf{i}}\right|^{2}\right\rangle} \ln \left(\frac{\left|\mathbf{E}_{\mathbf{i}}\right|^{2}}{\left\langle\left|\mathbf{E}_{\mathbf{i}}\right|^{2}\right\rangle}\right)\right\rangle+\frac{\left\langle\left|\mathbf{E}_{\mathbf{i}}\right|^{2}\right\rangle}{\left\langle\left|\mathbf{E}_{\mathbf{i}}\right|^{2}\right\rangle} \ln \left(\frac{1}{N}\right) \leq 0 \\
& \left\langle\frac{\left|\mathbf{E}_{\mathbf{i}}\right|^{2}}{\left\langle\left|\mathbf{E}_{\mathbf{i}}\right|^{2}\right\rangle} \ln \left(\frac{\left|\mathbf{E}_{\mathbf{i}}\right|^{2}}{\left\langle\left|\mathbf{E}_{\mathbf{i}}\right|^{2}\right\rangle}\right)\right\rangle-\ln (N) \leq 0 \\
& \left\langle\frac{\left|\mathbf{E}_{\mathbf{i}}\right|^{2}}{\left\langle\left|\mathbf{E}_{\mathbf{i}}\right|^{2}\right\rangle} \ln \left(\frac{\left|\mathbf{E}_{\mathbf{i}}\right|^{2}}{\left\langle\left|\mathbf{E}_{\mathbf{i}}\right|^{2}\right\rangle}\right)\right\rangle \leqslant \ln (N)
\end{aligned}
$$

It is also known from Kullback-Leibler divergence (or relative entropy) that:

$$
D_{K L}(p \| q)=\sum_{i} p_{i} \ln \left(\frac{p_{i}}{q_{i}}\right) \geqslant 0 \text { and } \sum_{i} p_{i}=1, \sum_{i} q_{i}=1
$$

Thus for $p_{i}=\frac{\left|\mathbf{E}_{\mathbf{i}}\right|^{2}}{\sum_{i}^{N}\left|\mathbf{E}_{\mathbf{i}}\right|^{2}}$ and taking a uniform distribution for $q$ with $q_{i}=\frac{1}{N}$ the following is true:

$$
\begin{aligned}
& \sum_{i}^{N} \frac{\left|\mathbf{E}_{\mathbf{i}}\right|^{2}}{\sum_{i}^{N}\left|\mathbf{E}_{\mathbf{i}}\right|^{2}} \ln \left(\frac{\left|\mathbf{E}_{\mathbf{i}}\right|^{2}}{\sum_{i}^{N}\left|\mathbf{E}_{\mathbf{i}}\right|^{2} \frac{1}{N}}\right) \geqslant 0 \\
& \frac{N}{N} \sum_{i}^{N} \frac{\left|\mathbf{E}_{\mathbf{i}}\right|^{2}}{\sum_{i}^{N}\left|\mathbf{E}_{\mathbf{i}}\right|^{2}} \ln \left(\frac{\left|\mathbf{E}_{\mathbf{i}}\right|^{2}}{\left\langle\left|\mathbf{E}_{\mathbf{i}}\right|^{2}\right\rangle}\right) \geqslant 0 \\
& \frac{1}{N} \sum_{i}^{N} \frac{\left|\mathbf{E}_{\mathbf{i}}\right|^{2}}{\sum_{i}^{N}\left|\mathbf{E}_{\mathbf{i}}\right|^{2} \frac{1}{N}} \ln \left(\frac{\left|\mathbf{E}_{\mathbf{i}}\right|^{2}}{\left\langle\left|\mathbf{E}_{\mathbf{i}}\right|^{2}\right\rangle}\right) \geqslant 0 \\
& \left\langle\frac{\left|\mathbf{E}_{\mathbf{i}}\right|^{2}}{\left\langle\left\langle\left.\mathbf{E}_{\mathbf{i}}\right|^{2}\right\rangle\right.} \ln \left(\frac{\left|\mathbf{E}_{\mathbf{i}}\right|^{2}}{\left\langle\left|\mathbf{E}_{\mathbf{i}}\right|^{2}\right\rangle}\right)\right\rangle \geqslant 0
\end{aligned}
$$

Therefore, with $\Delta I_{E}=\left\langle\frac{\left|\mathbf{E}_{\mathbf{i}}\right|^{2}}{\left\langle\left\langle\left.\mathbf{E}_{\mathbf{i}}\right|^{2}\right\rangle\right.} \ln \left(\frac{\left|\mathbf{E}_{\mathbf{i}}\right|^{2}}{\left\langle\left\langle\left.\mathbf{E}_{\mathbf{i}}\right|^{2}\right\rangle\right.}\right)\right\rangle$ :

$$
0 \leqslant \Delta I_{E} \leqslant \ln (N)
$$

The same is thus also valid for the negentropy in the time domain $\Delta I_{\epsilon}$.

\section{References}

[1] H.D.M. de Azevedo, A.M. Araújo, N. Bouchonneau, A review of wind turbine bearing condition monitoring: state of the art and challenges, Renew. Sustain. Energy Rev. 56 (2016) 368-379.

[2] B. Lu, Y. Li, X. Wu, Z. Yang, A review of recent advances in wind turbine condition monitoring and fault diagnosis, in: Power Electronics and Machines in Wind Applications, 2009. PEMWA 2009. IEEE, IEEE, 2009, pp. 1-7.

[3] P. Tchakoua, R. Wamkeue, M. Ouhrouche, F. Slaoui-Hasnaoui, T.A. Tameghe, G. Ekemb, Wind turbine condition monitoring: state-of-the-art review, new trends, and future challenges, Energies 7 (4) (2014) 2595-2630.

[4] R. Zimroz, W. Bartelmus, Gearbox condition estimation using cyclo-stationary properties of vibration signal, in: Key Engineering Materials, vol. 413, Trans Tech Publ, 2009, pp. 471-478.

[5] J. Antoni, J. Daniere, F. Guillet, Effective vibration analysis of IC engines using cyclostationarity. Part I-A methodology for condition monitoring, J. Sound Vibr. 257 (5) (2002) 815-837.

[6] R.B. Randall, Vibration-based Condition Monitoring: Industrial, Aerospace and Automotive Applications, John Wiley \& Sons, 2011. 
[7] J. Antoni, F. Bonnardot, A. Raad, M. El Badaoui, Cyclostationary modelling of rotating machine vibration signals, Mech. Syst. Signal Process. 18 (6) (2004) $1285-1314$.

[8] A. McCormick, A. Nandi, Cyclostationarity in rotating machine vibrations, Mech. Syst. Signal Process. 12 (2) (1998) $225-242$.

[9] I. Antoniadis, G. Glossiotis, Cyclostationary analysis of rolling-element bearing vibration signals, J. Sound Vibr. 248 (5) (2001) $829-845$.

[10] C. Capdessus, M. Sidahmed, J. Lacoume, Cyclostationary processes: application in gear faults early diagnosis, Mech. Syst. Signal Process. 14 (3) (2000) $371-385$.

[11] P. Borghesani, P. Pennacchi, R. Ricci, S. Chatterton, Testing second order cyclostationarity in the squared envelope spectrum of non-white vibration signals, Mech. Syst. Signal Process. 40 (1) (2013) 38-55.

[12] J. Antoni, G. Xin, N. Hamzaoui, Fast computation of the spectral correlation, Mech. Syst. Signal Process. 92 (2017) $248-277$.

[13] R.B. Randall, J. Antoni, S. Chobsaard, The relationship between spectral correlation and envelope analysis in the diagnostics of bearing faults and other cyclostationary machine signals, Mech. Syst. Signal Process. 15 (5) (2001) 945-962.

[14] R.B. Randall, J. Antoni, Rolling element bearing diagnostics - a tutorial, Mech. Syst. Signal Process. 25 (2) (2011) $485-520$.

[15] E. Candes, J. Romberg, T. Tao, Robust uncertainty principles: exact signal reconstruction from highly incomplete frequency information, arXiv preprint math/0409186.

[16] E. Candes, T. Tao, Near optimal signal recovery from random projections: Universal encoding strategies? arXiv preprint math/0410542.

[17] D.L. Donoho et al, Compressed sensing, IEEE Trans. Inf. Theory 52 (4) (2006) 1289-1306.

[18] D.L. Donoho, For most large underdetermined systems of linear equations the minimal l1-norm solution is also the sparsest solution, Commun. Pure Appl. Math.: J. Issued by the Courant Institute of Mathematical Sciences 59 (6) (2006) 797-829.

[19] Web of science research records for sparsity,http://wcs.webofknowledge.com/RA/analyze.do?product=WOS\&SID=E6K2nUlrn1a36enVBn3\&field=PY PublicationYear_PublicationYear_en\&yearSort=true (accessed: 2019-03-12).

[20] R.A. Wiggins, Minimum entropy deconvolution, Geoexploration 16 (1-2) (1978) 21-35.

[21] J. Antoni, The Infogram: entropic evidence of the signature of repetitive transients, Mech. Syst. Signal Process. 74 (2016) $73-94$.

[22] C.A. Cabrelli, Minimum entropy deconvolution and simplicity: a noniterative algorithm, Geophysics 50 (3) (1985) $394-413$.

[23] C.-S. Wang, J. Tang, B. Zhao, An improvement on d norm deconvolution: a fast algorithm and the related procedure, Geophysics 56 (5) (1991) 675-680.

[24] W.C. Gray, Variable norm deconvolution, no. 19, Stanford University Ph. D. thesis, 1979.

[25] G.L. McDonald, Q. Zhao, Multipoint optimal minimum entropy deconvolution and convolution fix: application to vibration fault detection, Mech. Syst. Signal Process. 82 (2017) 461-477.

[26] J. Obuchowski, R. Zimroz, A. Wyłomańska, Blind equalization using combined skewness-kurtosis criterion for gearbox vibration enhancement, Measurement 88 (2016) 34-44.

[27] H. Endo, R. Randall, Enhancement of autoregressive model based gear tooth fault detection technique by the use of minimum entropy deconvolution filter, Mech. Syst. Signal Process. 21 (2) (2007) 906-919.

[28] R.J.G. de Oliveira, M.H. Mathias, Maximization of the signal impulsiveness combining envelope technique with minimum entropy deconvolution, in: Applied Mechanics and Materials, vol. 392, Trans Tech Publ, 2013, pp. 725-729.

[29] H. Endo, R. Randall, C. Gosselin, Differential diagnosis of spall vs. cracks in the gear tooth fillet region: experimental validation, Mech. Syst. Signal Process. 23 (3) (2009) 636-651.

[30] T. Barszcz, N. Sawalhi, Fault detection enhancement in rolling element bearings using the minimum entropy deconvolution, Arch. Acoustics 37 (2) (2012) 131-141.

[31] D.-H. Kwak, D.-H. Lee, J.-H. Ahn, B.-H. Koh, Fault detection of roller-bearings using signal processing and optimization algorithms, Sensors 14 (1) (2014) $283-298$.

[32] J. Antoni, Cyclic spectral analysis in practice, Mech. Syst. Signal Process. 21 (2) (2007) 597-630.

[33] W.A. Smith, R.B. Randall, X.D.C. du Mée, P. Peng, Use of cyclostationary properties to diagnose planet bearing faults in variable speed conditions, in: 10th DST group international conference on health and usage monitoring systems, 17th Australian aerospace congress, 2017 , pp. 26-28.

[34] J. Antoni, Cyclic spectral analysis of rolling-element bearing signals: facts and fictions, J. Sound Vibr. 304 (3-5) (2007) 497-529.

[35] M. Buzzoni, J. Antoni, G. D’Elia, Blind deconvolution based on cyclostationarity maximization and its application to fault identification, J. Sound Vib. 432 (2018) 569-601.

[36] M. Wang, Z. Mo, H. Fu, H. Yu, Q. Miao, Harmonic 12/11 norm for bearing fault diagnosis, IEEE Access.

[37] A. Repetti, M.Q. Pham, L. Duval, E. Chouzenoux, J.-C. Pesquet, Euclid in a taxicab: sparse blind deconvolution with smoothed 11/12 regularization, IEEE Signal Process. Lett. 22 (5) (2015) 539-543.

[38] M. Zibulevsky, B.A. Pearlmutter, Blind source separation by sparse decomposition in a signal dictionary, Neural Comput. 13 (4) (2001) 863-882.

[39] B. Barak, J.A. Kelner, D. Steurer, Rounding sum-of-squares relaxations, in: Proceedings of the forty-sixth annual ACM symposium on Theory of computing, ACM, 2014, pp. 31-40.

[40] M. Morup, K.H. Madsen, L.K. Hansen, Approximate 10 constrained non-negative matrix and tensor factorization, in: 2008 IEEE International Symposium on Circuits and Systems, IEEE, 2008, pp. 1328-1331.

[41] N. Hurley, S. Rickard, Comparing measures of sparsity, IEEE Trans. Inf. Theory 55 (10) (2009) $4723-4741$.

[42] P.O. Hoyer, Non-negative matrix factorization with sparseness constraints, J. Mach. Learn. Res. 5 (Nov) (2004) 1457-1469.

[43] X. Jia, M. Zhao, Y. Di, P. Li, J. Lee, Sparse filtering with the generalized lp/lq norm and its applications to the condition monitoring of rotating machinery, Mech. Syst. Signal Process. 102 (2018) 198-213.

[44] R.A. Hom, C.R. Johnson, Matrix analysis, Cambridge University Express.

[45] R. Mises, H. Pollaczek-Geiringer, Praktische verfahren der gleichungsauflösung, ZAMM-Journal of Applied Mathematics and Mechanics/Zeitschrift für Angewandte Mathematik und Mechanik 9 (2) (1929) 152-164.

[46] E. Bechhoefer, M. Kingsley, A review of time synchronous average algorithms, in: Annual conference of the prognostics and health management society, vol. 1, 2009, pp. 1-10.

[47] C. Peeters, P. Guillaume, J. Helsen, A comparison of cepstral editing methods as signal pre-processing techniques for vibration-based bearing fault detection, Mech. Syst. Signal Process. 91 (2017) 354-381.

[48] R. Randall, N. Sawalhi, Editing time signals using the real cepstrum, in: MFPT Conference, Virginia Beach (May 2011 ), 2011.

[49] J. Antoni, R. Randall, Unsupervised noise cancellation for vibration signals: part ii a novel frequency domain algorithm, Mech. Syst. Signal Process. 18 (1) (2004) 103-117.

[50] J. Antoni, R. Randall, Unsupervised noise cancellation for vibration signals: part i evaluation of adaptive algorithms, Mech. Syst. Signal Process. 18 (1) (2004) 89-101.

[51] P. Borghesani, P. Pennacchi, S. Chatterton, R. Ricci, The velocity synchronous discrete fourier transform for order tracking in the field of rotating machinery, Mech. Syst. Signal Process. 44 (1-2) (2014) 118-133.

[52] J. Lee, H. Qiu, G. Yu, J. Lin, Rexnord technical services: Bearing data set, Moffett Field, CA: IMS, Univ. Cincinnati. NASA Ames Prognostics Data Repository, NASA Ames.

[53] H. Qiu, J. Lee, J. Lin, G. Yu, Wavelet filter-based weak signature detection method and its application on rolling element bearing prognostics, J. Sound Vibr. 289 (4-5) (2006) 1066-1090.

[54] J. Antoni, P. Borghesani, A statistical methodology for the design of condition indicators, Mech. Syst. Signal Process. 114 (2019) $290-327$. 